\title{
Alzheimer's genetic risk factor FERMT2 (Kindlin-2) controls axonal growth and synaptic plasticity in an APP-dependent manner
}

\author{
Fanny Eysert ${ }^{1} \cdot$ Audrey Coulon $^{1} \cdot$ Emmanuelle Boscher ${ }^{2,3} \cdot$ Anaïs-Camille Vreulx $^{1} \cdot$ Amandine Flaig $^{1}$. \\ Tiago Mendes ${ }^{1} \cdot$ Sandrine Hughes $^{4} \cdot$ Benjamin Grenier-Boley $^{1} \cdot$ Xavier Hanoulle $^{5}$ - Florie Demiautte ${ }^{1}$. \\ Charlotte Bauer ${ }^{6} \cdot$ Mikael Marttinen $\mathbb{D}^{7} \cdot$ Mari Takalo $^{7} \cdot$ Philippe Amouyel $\mathbb{D}^{1} \cdot$ Shruti Desai $^{1} \cdot$ Ian Pike ${ }^{8}$. \\ Mikko Hiltunen $^{7} \cdot$ Frédéric Chécler $^{6} \cdot$ Mélissa Farinelli $^{4} \cdot$ Charlotte Delay $^{1} \cdot$ Nicolas Malmanche $^{1}$. \\ Sébastien S. Hébert ${ }^{2,3} \cdot$ Julie Dumont $^{1} \cdot$ Devrim Kilinc $^{1} \cdot$ Jean-Charles Lambert $\mathbb{B}^{1} \cdot$ Julien Chapuis $\mathbb{D}^{1}$
}

Received: 12 December 2019 / Revised: 2 October 2020 / Accepted: 19 October 2020 / Published online: 3 November 2020

(c) The Author(s) 2020. This article is published with open access, corrected publication 2020

\begin{abstract}
Although APP metabolism is being intensively investigated, a large fraction of its modulators is yet to be characterized. In this context, we combined two genome-wide high-content screenings to assess the functional impact of miRNAs and genes on APP metabolism and the signaling pathways involved. This approach highlighted the involvement of FERMT2 (or Kindlin-2), a genetic risk factor of Alzheimer's disease (AD), as a potential key modulator of axon guidance, a neuronal process that depends on the regulation of APP metabolism. We found that FERMT2 directly interacts with APP to modulate its metabolism, and that FERMT2 underexpression impacts axonal growth, synaptic connectivity, and long-term potentiation in an APP-dependent manner. Last, the rs7143400-T allele, which is associated with an increased AD risk and localized within the 3'UTR of FERMT2, induced a downregulation of FERMT2 expression through binding of miR-4504 among others. This miRNA is mainly expressed in neurons and significantly overexpressed in AD brains compared to controls. Altogether, our data provide strong evidence for a detrimental effect of FERMT2 underexpression in neurons and insight into how this may influence AD pathogenesis.
\end{abstract}

These authors contributed equally: Jean-Charles Lambert, Julien Chapuis

Supplementary information The online version of this article (https:// doi.org/10.1038/s41380-020-00926-w) contains supplementary material, which is available to authorized users.

Julien Chapuis

julien.chapuis@pasteur-lille.fr

1 Université de Lille, Inserm, CHU Lille, Institut Pasteur de Lille, U1167-RID-AGE-Facteurs de Risque et Déterminants Moléculaires des Maladies Liées au Vieillissement, Lille 59019, France

2 Centre de Recherche du CHU de Québec-Université Laval, CHUL, Axe Neurosciences, Québec City, QC, Canada

3 Faculté de Médecine, Département de Psychiatrie et de Neurosciences, Université Laval, Québec City, QC, Canada

\section{Introduction}

$\mathrm{AD}$ is a neurodegenerative disease characterized by two main pathological hallmarks: (i) intracellular neurofibrillary tangles consisting of hyperphosphorylated Tau proteins and (ii) extracellular amyloid plaques consisting of aggregates of $\beta$-amyloid (A $\beta)$ peptides resulting from the processing of amyloid precursor protein (APP). Three main proteases $(\alpha-, \beta-$, and $\gamma$-secretases) are involved in APP processing through (i) the amyloidogenic pathway

4 E-Phy-Science, Bioparc de Sophia Antipolis, 2400 route des Colles, Biot 06410, France

5 Université de Lille, CNRS, UMR8576-Labex DISTALZ, Villeneuve d'Ascq 59655, France

6 Université Côte d'Azur, Inserm, CNRS, IPMC, DistAlz Laboratory of Excellence, Valbonne, France

7 Institute of Biomedicine, University of Eastern Finland, Kuopio, Finland

8 Proteome Sciences plc, Hamilton House, London WC1H 9BB, UK 
( $\beta$ - and $\gamma$-secretases), leading to $A \beta$ production, and (ii) the non-amyloidogenic pathway ( $\alpha$ - and $\gamma$-secretases), which prevents $A \beta$ generation by cleaving APP within the $\mathrm{A} \beta$ sequence [1].

The identification of early-onset autosomal-dominant AD-linked mutations in the genes for $A P P$ and presenilins (PSEN1 and PSEN2, part of the $\gamma$-secretase), has placed abnormal APP metabolism at the center of the disease, further supporting the amyloid-cascade hypothesis $[2,3]$ : the overproduction of $A \beta$ peptides-especially the longer forms that are thought to be more neurotoxic-may lead to (or favor) Tau pathology and subsequent neuronal death.

Although the validity of the amyloid-cascade hypothesis is debated [4], the importance of APP has recently been emphasized by the discovery of a rare APP mutation hampering $A \beta$ production that lowers $A D$ risk [5]. Moreover, loss-of-function variants in Sortilin-related receptor (L(DLR class A)), which is a strong regulator of APP metabolism and $A \beta$ production, are associated with earlyand late-onset forms of $\mathrm{AD}$ [6-8]. Beyond $\mathrm{A} \beta$ production, the involvement of genetic risk factors, such as APOE and TREM 2 in modulation of $A \beta$ aggregation and/or degradation/clearance, has been proposed to be essential in the $\mathrm{AD}$ process $[9,10]$. Recent high-throughput genomic approaches have also highlighted APP metabolism in the AD pathophysiological process: the main actors of APP metabolism, e.g., ADAM10 and APH1B (part of the $\gamma$-secretase complex), have been characterized as genetic determinants $[11,12]$, and numerous other genetic determinants have been described as potential modulators of APP metabolism (for a review, see ref. [13]).

Among these genetic determinants, FERMT2 has been identified to be involved in APP metabolism using an agnostic, systematic approach, i.e., high-content screening of 18,107 siRNA pools in HEK293 cells stably overexpressing an APP fusion protein (mCherry-APP ${ }^{695 \mathrm{WT}}$ YFP) that allows for the quantification of intracellular APP fragments [14]. Following this initial screening, FERMT2 underexpression was then specifically associated with increasing levels of mature APP at the cell surface, where FERMT2 facilitates APP recycling, resulting in increased A $\beta$-peptide production [14].

Little is known about FERMT2. This protein localizes to focal adhesions, where it is proposed to interact with $\beta 3$ integrin and to be a major actor in integrin activation [15]. FERMT2 has been reported as a key protein involved in cardiac and skeletal muscle development [16], and has been involved in cancer progression [17-19]. However, despite the fact that FERMT2 is a genetic risk factor of $\mathrm{AD}$, the physiological and/or pathophysiological roles of FERMT2 in the brain have not been identified. Within this background, we sought to determine how FERMT2 regulation impacts APP metabolism and/or AD risk and its involvement in neuronal functions.

\section{Methods}

\section{Cell culture}

Human HeLa (RRID:CVCL_0030) and HEK293 (RRID: CVCL_0045) cells were respectively maintained in Eagle's minimal essential medium (American Type Culture Collection, Teddington, UK) and DMEM/Ham's F-12 1:1 medium (Life Technologies, Carlsbad, CA) supplemented with $10 \%$ heat-inactivated fetal bovine serum and $2-\mathrm{mM} \mathrm{L}_{\mathrm{L}}$ glutamine, penicillin $(10 \mathrm{UI} / \mathrm{mL}) /$ streptomycin $(10 \mu \mathrm{g} / \mathrm{mL})$. All cell lines were tested negative for mycoplasma contamination using PCR test (Venor GeM OneStep, Minerva Biolabs, Germany).

\section{Microfluidic chip fabrication}

Masters of multicompartment microfluidic devices were fabricated through photolithography as previously described [20]. Polydimethylsiloxane (PDMS, Sylgard 184, Dow Corning, Midland, MI) pads were replica-molded $(2 \mathrm{~h}$ at $70{ }^{\circ} \mathrm{C}$ ) and irreversibly bonded to glass coverslips via $\mathrm{O}_{2}$ plasma (Diener, Ebhausen, Germany). The devices were placed in plastic Petri dishes, wetted with $\mathrm{dH}_{2} \mathrm{O}$, and UVsterilized for $30 \mathrm{~min}$.

\section{Primary neuronal culture and viral transductions}

Animal housing and experimentation were carried out in accordance with the French Council in Animal Care guidelines for the care and use of animals and following the protocols approved by the Institut Pasteur de Lille ethical committee. Primary neuronal cultures were obtained from hippocampus or cortices of postnatal (P0) rats as described previously [21]. Briefly, after the dissection of the brains, hippocampi were washed three times in HBSS (HBSS, 1-M HEPES, penicillin/streptomycin, and 100-mM sodium pyruvate, Gibco) and were dissociated via trypsin digestion $\left(2.5 \%, 37^{\circ} \mathrm{C}\right.$, Gibco) for $7 \mathrm{~min}$. Next, hippocampi were incubated with DNAse $(5 \mathrm{mg} / \mathrm{mL}$, Sigma) for $1 \mathrm{~min}$ and washed again in MEM medium supplemented with $10 \%$ SVF, $1 \%$ Glutamax, $0.8 \%$ MEM vitamins, $0.5 \%$ penicillin/ streptomycin, and $0.45 \%$ D-glucose (Sigma). With a pipette, hippocampi were mechanically dissociated and resuspended in Neurobasal A medium (Gibco) supplemented with $2 \%$ B27 (Gibco) and 0.25\% GlutaMax. In total, 200,000 neurons were seeded per well in 24-well plates. In total, 50,000 neurons were seeded in the somatic chamber of microfluidic devices, precoated with poly-L-lysine $(0.1 \mathrm{mg} / \mathrm{mL}$, Sigma $)$ 
in borate buffer $(0.31 \%$ boric acid, $0.475 \%$ sodium tetraborate, $\mathrm{pH}=8.5$ ). About $0.1 \%$ EDTA (in $\mathrm{H}_{2} \mathrm{O}$ ) was added to the Petri dishes containing microfluidic devices to minimize evaporation. The culture medium was refreshed every 3 days. Neurons were maintained at $37^{\circ} \mathrm{C}$ in a humidified $5 \% \mathrm{CO}_{2}$ incubator.

\section{Lentiviral transductions}

Lentiviral transductions were carried out at 1 day in vitro (DIV1) with a multiplicity of infection (MOI) of 10 . In the case of co-transduction, MOI of 5 was used for each lentivirus. Briefly, lentiviruses were diluted in culture medium containing $4-\mu \mathrm{g} / \mathrm{mL}$ polybrene (hexadimethrine bromide, Sigma) and were added to the cells. After $6 \mathrm{~h}$ of transduction, lentivirus suspension was replaced with fresh medium. The following lentiviruses were used for transduction: Mission shRNA vectors (Sigma) shNT (Non-Mammalian shRNA Control, SHC002), shFERMT2 (TRCN0000191859), shAPP (TRCN0000006707), and pLenti6 empty vectors (Mock) or including human FERMT2 ${ }^{\mathrm{WT}}$ or FERMT2 $^{\mathrm{QW}}$ cDNA sequences. LifeAct-Ruby lentivirus (pLenti.PGK.LifeActRuby.W: RRID:Addgene_51009) was a kind gift from Rusty Lansford.

\section{RFLP genotyping}

Genomic DNA in the vicinity of the rs7143400 was amplified by PCR using the following primers $5^{\prime}$-GGTT GGGTGTGAATAGGAAT- $3^{\prime}$ and 5'-TGCATGCCTGATT $^{\prime}$ TATTTGG-3' $3^{\prime}$ before digestion with Tsp45I enzyme (Thermo Scientific). Finally, treated PCR products were analyzed in $2 \%$ agarose gel to visualize the cleavage bands.

\section{Designing CRISPR/Cas9 and genome editing}

gRNA sequences were predicted by Benchling (http://www. benchling.com) and cloned into the GeneArt CRISPR OFP Nuclease Vector (ThermoFisher Scientific) allowing Cas9 and gRNA expression. Homology-directed repair was induced by co-transfection of 71-pb double-strained DNA oligonucleotide template, including rs7143400-T allele in HEK293 cells (Supplementary Fig. 1). HEK293 clones were isolated by limiting dilution before RFLP genotyping. Sequence integrity of the FERMT2 $3^{\prime} \mathrm{UTR}$ and predicted potential off-target sites were validated by Sanger sequencing (Supplementary Fig. 1).

\section{Visualization of miRNA expression at the single-cell level}

To visualize RNA molecules by fluorescence at single-cell resolution and quantify gene expression, we used
ViewRNA Cell Plus Assay kit (ThermoFisher Scientific) according to the manufacturer's instructions. Briefly, after fixation and permeabilization, cells were washed $3 \times$ with PBS containing RNAse inhibitor and were incubated with probes directed against specific mRNA or miRNA for $2 \mathrm{~h}$ at $40{ }^{\circ} \mathrm{C}$. After washes, probes were amplified, first, in a preamplifier solution, and second, in an amplifier solution, both for $1 \mathrm{~h}$ at $40^{\circ} \mathrm{C}$. Then, cells were incubated with nucleotide probes stained with different fluorophores, allowing the detection of mRNA or miRNA puncta. This approach was coupled with immunofluorescence experiments described.

\section{miRNA quantification in human brain samples}

This study was approved by CHU de Québec-Université Laval Research Ethics Committee (\#2017-3017). Frozen human brain tissue $(0.5-1.2 \mathrm{~g}$ per sample) was obtained from the Harvard Brain Tissue Resource Center in Belmont, USA, the Brain Endowment Bank in Florida, USA, and the Human Brain and Spinal Fluid Resource Center in Los Angeles, USA, via NIH Neurobiobank. The cohort of patients included nondementia controls $(n=30)$ and $\mathrm{AD}$ cases $(n=52)$ based on neuropathological diagnosis. Upon receipt of the specimens, frozen postmortem parietal cortex (BA39) was crushed using a biopulverizer prior to RNA extraction and analysis.

Total RNA was extracted from brain tissue (Supplementary Table 1) using TRIzol reagent (Ambion, 15596018) according to the manufacturer's instructions. miRNA quantifications were done using the TaqMan miR Reverse Transcription Kit (Applied Biosystem, Burlington, Canada) and TaqMan Universal Master Mix (Applied Biosystem, 4324018) following the manufacturer's instructions. Primers were purchased from ThermoFisher (miR-4504 ID: 464271_mat; RNU48 ID: 001006; miR-222-3p ID: 002276). MiR-4504 and miR-222 levels were normalized to RNU48. The relative amounts of each mature miRNA were calculated using the comparative $\mathrm{Ct}\left(2^{-\Delta \Delta \mathrm{Ct}}\right)$ method [22].

\section{Immunoblotting and $A \boldsymbol{\beta}$ quantification}

Equal amounts (10-25 $\mu \mathrm{g})$ of cell lysate were collected in RIPA buffer (1-M Tris, $1.5-\mathrm{M} \mathrm{NaCl}, 0.1 \%$ NP-40, $10 \%$ SDS, $100-\mathrm{mM}$ sodium orthovanadate, and $0.5 \%$ sodium deoxycholate, $\mathrm{pH}=7.4$ ) containing protease inhibitors (Complete mini, Roche Applied Science, Penzberg, Germany), lithium dodecyl sulfate (LDS), and reducing agent (Invitrogen). Samples were denaturated and analyzed using SDS-PAGE and the following antibodies: human FERMT2 (RRID:AB_10727911), APP C-terminal domain (RRID: AB_258409), actin (RRID:AB_476692), A $\beta$ clone 6E10 (RRID:AB_662798), A $\beta$ clone 4G8 (RRID:AB_662812), Synaptophysin I (RRID:AB_887824), PSD95 (RRID: 
AB_2619800), and GAPDH (RRID:AB_10615768). Extracellular culture media were collected in order to dosesecrete $\mathrm{A} \beta$ using Alpha-LISA assays (Alpha-LISA Amyloid $\beta_{1-\mathrm{X}}$ Kit, AL288C, PerkinElmer) according to the manufacturer's instructions.

\section{Cell-surface biotinylation}

HEK293-APP ${ }^{695 W T}$ cells were transfected with PCDNA4.1, FERMT2 $^{\text {WT }}$, or FERMT2 ${ }^{\mathrm{QW}}$ (PCDNA4/HisMax, Thermo Scientific V86420) for $48 \mathrm{~h}$. Next, cell-surface proteins were biotinylated using sulfo-NHS-SS-biotin (sulfosuccinimidyl20(biotinamido)ethyl-1,3-dithiopropionate) for $30 \mathrm{~min}$ at $4{ }^{\circ} \mathrm{C}$ according to the manufacturer's instructions (Cell Surface Protein Isolation Kit, Pierce, 89881). Then, cells were lysed and immunoprecipitated with streptavidin beads. Precipitated proteins were eluted from the beads with loading buffer containing 50-mM DTT, heated for $10 \mathrm{~min}$ at $95^{\circ} \mathrm{C}$, and analyzed by WB.

\section{Co-immunoprecipitation}

Equal amounts of protein were collected in coimmunoprecipitation buffer (10-mM HEPES, 140-mM $\mathrm{NaCl}$, and $0.5 \% \mathrm{NP}-40, \mathrm{pH}=7.4)$ containing protease inhibitors (Complete mini, Roche Applied Science) and phosphatase inhibitor (100-mM sodium orthovanadate) and incubated with the primary $A \beta$ antibody clone 4G8 (RRID:AB_662812) overnight, with gentle rocking. Production of recombinant $\mathrm{C} 100$ fragment was performed as previously described [23]. Co-immunoprecipitation was carried out using Pierce Protein A/G magnetic beads kit (Thermo Scientific, 88802) according to the manufacturer's instructions. Samples with proteins and antibody complexes were incubated with $25 \mu \mathrm{L}(0.25 \mathrm{mg})$ of A/G magnetic beads previously washed with coimmunoprecipitation buffer. After $1 \mathrm{~h}$ of incubation at $4{ }^{\circ} \mathrm{C}$, the magnetic beads were washed $3 \times$, resuspended with loading buffer (LDS and reducing agent) for $10 \mathrm{~min}$ at RT, and analyzed by WB.

\section{Immunofluorescence and PLA}

Cells were fixed in $4 \%$ paraformaldehyde (PFA) for $15 \mathrm{~min}$, washed $3 \times$ with PBS, and permeabilized for 5 min with $0.3 \%$ Triton X-100. Cells were incubated with 5\% normal donkey serum for $2 \mathrm{~h}$ at RT before overnight incubation with the following primary antibodies: human FERMT2 (RRID: AB_10727911), Kindlin-2 (RRID:AB_2278298), APP Cterminal domain (RRID:AB_258409), APP A4 clone 22C11 (RRID:AB_94882), Synaptophysin I (RRID:AB_887824), PSD95 (RRID:AB_2619800), Homer (RRID:AB_2631222), and $\alpha$-Tubulin (RRID:AB_2210391). The cells were then washed $3 \times$ with PBS and incubated with the following secondary antibodies raised in donkey (AlexaFluor-conjugated AffiniPure Fragment 405, 488, 594, or 647, Jackson ImmunoResearch), 1:10,000 Hoechst 33342, or 1/40 SiR-Actin probe (SC001, Spirochrome). Alternatively, Kindlin-2 (RRID:AB_2278298) and APP A4 22C11 (RRID: AB_94882) antibodies were used for the proximity-ligation assay (PLA) according to the manufacturer's instructions (Duolink ${ }^{\circ}$, Olink Bioscience).

\section{Live-cell microscopy for axon elongation and actin dynamics}

After DIV5, once the axons reached the axonal chamber of microfluidic devices, the culture medium was replaced with Neurobasal A without phenol red, supplemented with GlutaMax, $2 \% \mathrm{~B}_{27}$, and 25-mM HEPES. Phase-contrast images of growing axons were acquired every $10 \mathrm{~min}$ for 110 min using Zeiss AxioObserver Z1 microscope equipped with a Prime 95B Scientific CMOS (Photometrics, Tucson, AZ) camera and $32 \times$ objective. Movies were analyzed using Fiji MTrack J Plugin [24] to determine the axongrowth speed.

To visualize filamentous actin (F-actin) dynamics in the growth cones of elongating axons, neurons were cotransducted with LifeAct-Ruby at DIV1. At DIV5, growth cones expressing LifeAct-Ruby were imaged using a Nikon microscope equipped with Yokogawa spinning-disk system and a Nikon CFI Apochromat 100x TIRF objective (NA $1.49)$, in live superresolution mode $(66 \mathrm{~nm} / \mathrm{px})$. Processed movies were analyzed using Imaris (Bitplane, Zurich, Switzerland) surface tracking tool to obtain the speed and direction of F-actin puncta undergoing actinretrograde flow.

\section{Synaptosome extraction}

To verify the presence of proteins at the synaptic level, we did a subcellular fractionation as previously described [25]. Briefly, cortical neurons were resuspended in a solution (0.32-M sucrose and 10-mM HEPES, $\mathrm{pH}=7.4)$ and were centrifuged at $1000 \times g$ for $10 \mathrm{~min}$ to remove nuclei and debris. The supernatant was centrifuged at $12,000 \times g$ for $20 \mathrm{~min}$ to remove the cytosolic fraction. The pellet was resuspended in a second solution (4-mM HEPES, 1-mM EDTA, $\mathrm{pH}=7.4)$ and was centrifuged $2 \times$ at $12,000 \times g$ for $20 \mathrm{~min}$. The new pellet was resuspended in a third solution (20-mM HEPES, $100-\mathrm{mM} \mathrm{NaCl}$, and $0.5 \%$ Triton X-100, $\mathrm{pH}=7.2$ ) for $1 \mathrm{~h}$ at $4{ }^{\circ} \mathrm{C}$ and centrifuged at $12,000 \times g$ for $20 \mathrm{~min}$. The supernatant collected corresponds to the nonPSD fraction (Triton-soluble). The remaining pellet was resuspended in a fourth solution (20-mM HEPES, 0.15-mM $\mathrm{NaCl}, 1 \%$ Triton $\mathrm{X}-100,1 \%$ deoxycholicacid, and $1 \%$ SDS, 
$\mathrm{pH}=7.5$ ) for $1 \mathrm{~h}$ at $4{ }^{\circ} \mathrm{C}$ and was centrifuged at $10,000 \times g$ for $15 \mathrm{~min}$ to obtain a supernatant containing the PSD fraction (Triton-insoluble). The different fractions were then analyzed by WB.

\section{Quantification of synaptic connectivity}

To quantify synaptic connectivity, we transducted primary hippocampal neurons in pre- and/or postsynaptic compartments of microfludic devices at DIV1 with lentiviruses carrying shNT and/or shFERMT2 $(\mathrm{MOI}=10)$. At DIV14, cultures were fixed and immunostained against Synaptophysin I and Homer pre- and postsynaptic markers, respectively. Synaptic compartments were imaged with Zeiss LSM880 confocal microscope, using a $63 \times 1.4-\mathrm{NA}$ objective and the AiryScan superresolution unit. Images were analyzed with Imaris software (Bitplane, Zürich, Switzerland) by reconstructing Synaptophysin I and Homer puncta in 3D. The volume and position information of all puncta were processed using a custom Matlab (MathWorks, Natick, MA) program. This program assigns each postsynaptic spot to the nearest presynaptic spot (within a distance threshold of $1 \mu \mathrm{m}$ ) and calculates the number of such assignments for all presynaptic puncta. The percentage of presynaptic spots not assigned by any postsynaptic spot was consistently used as a readout of synaptic connectivity [26].

\section{Lentivirus injection}

For stereotactic injections, C57B16/J mice (RRID: IMSR_JAX:000664) were anesthetized with 4\% isoflurane $(2 \mathrm{~L} / \mathrm{min})$ and placed in a stereotaxic frame (68528, RWD Life Science, Shenzhen, China) in which the head of the animal was fixed with a pair of ear bars and a perpendicular tooth bar. During surgical procedures, $1.5 \%$ isoflurane $(2 \mathrm{~L} / \mathrm{min})$ was delivered through a facial mask via spontaneous respiration. Their body temperature was maintained between 36.5 and $37.5^{\circ} \mathrm{C}$ with a homeothermic blanket. The head was shaved and Vetedine was applied. Wounds and pressure points were infiltrated with lidocaine. A skin incision was made along the sagittal midline of the scalp. Craniotomy was made to target the structures of interest. Lentiviruses were injected into the right and left hippocampus $(1.5 \mu \mathrm{L}$ per hemisphere, $0.2 \mu \mathrm{L} / \mathrm{min}$ ). After injections, wound clips were used for skin closure. For the sham group, surgical procedures were performed without any injection. During the surgery, the level of anesthesia was regularly verified by testing the nociceptive hind-limb withdrawal reflex. Subjects were then allowed to recover in their home cages for at least 7 days before sacrifice for ex vivo electrophysiological recordings.

\section{Hippocampal acute-slice preparation}

One week after the surgery, sagittal hippocampal brain slices were obtained using standard brain-slicing methods. Mice were deeply anesthetized with isoflurane and decapitated. The brain was quickly removed and immersed in icecold preoxygenated artificial cerebrospinal fluid (aCSF) containing 124-mM NaCl, 3.75-mM KCl, 2- $\mathrm{mM} \mathrm{MgSO}_{4}$, 2-

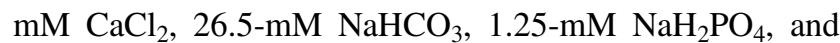
$10-\mathrm{mM}$ glucose, and was continuously oxygenated $(\mathrm{pH}=$ 7.4, $27^{\circ} \mathrm{C}$ ). In all, 350- $\mu$ m-thick slices were prepared using a Vibratome (VT 1000S, Leica Microsystems, Bannockburn, IL), and placed in a holding chamber filled with aCSF. Slices were allowed to recover in these conditions at least 1 $\mathrm{h}$ before recording.

\section{Electrophysiological recordings}

For electrophysiological recordings, a single slice was placed in the recording chamber, submerged and continuously superfused with gassed $\left(95 \% \mathrm{O}_{2}\right.$ and $\left.5 \% \mathrm{CO}_{2}\right)$ aCSF at a constant rate $(2 \mathrm{~mL} / \mathrm{min})$. Extracellular field excitatory postsynaptic potentials (fEPSPs) were recorded in the CA1 stratum radiatum using a glass micropipette filled with aCSF. fEPSPs were evoked by the electrical stimulation of Schaffer collaterals/commissural pathway at $0.1 \mathrm{~Hz}$ with a glass-stimulating electrode placed in the stratum radiatum (100- $\mu$ s duration).

To test the effect of miRNA-expressing lentiviruses on basal synaptic transmission, input/output (I/V) curves were constructed at the beginning of the experiment. The slope of fEPSPs was measured and plotted against different intensities of stimulation (from 0 to $100 \mu \mathrm{A}$ ).

Stable baseline fEPSPs were recorded by stimulating at $30 \%$ maximal field amplitude for $10 \mathrm{~min}$ prior to the beginning of the experiment (single-pulse stimulation every $10 \mathrm{~s}(0.1 \mathrm{~Hz}))$. The same intensity of stimulation was kept for the remainder of the experiment. For the paired-pulse facilitation (PPF) protocol, two stimulations were applied with 50-, 100-, 150-, 200-, 300-, 400-, and 500-ms interval. For long-term potentiation (LTP) protocol, after a 10-min stable baseline period, LTP was induced by the following stimulation protocol: three trains of 100 stimulations at $100 \mathrm{~Hz}$ at the same stimulus intensity, with 20 -s intervals between trains. Following this conditioning stimulus, a 1-h test period was recorded where responses were again elicited by a single-pulse stimulation every $10 \mathrm{~s}(0.1 \mathrm{~Hz})$ at the same stimulus intensity. Signals were amplified with an Axopatch 200B amplifier (Molecular Devices, Union City, CA) digitized by a Digidata 1550 interface (Axon Instruments, Molecular Devices, San Jose, CA) and sampled at $10 \mathrm{kHz}$. Recordings were acquired using Clampex (Molecular Devices) and analyzed with Clampfit (Molecular 
Devices). Experimenters were blinded to treatment for all experiments.

\section{Statistical analysis}

No statistical methods were used to predetermine sample sizes; sample sizes were determined according to data reported in previous publications. All the results are presented as mean \pm SD. Statistically significant outliers were calculated and excluded, using the mean absolute deviation (MAD) method ( \pm 3 MAD). Data were analyzed either using nonparametric (Mann-Whitney) test or a two-way ANOVA (Kruskal-Wallis) for multiple comparisons, including Bonferroni's correction, as indicated in the figure legends. For electrophysiological recordings, unpaired t tests were used to compare groups of hippocampal slides. Data analysis was not blinded.

\section{Results}

\section{FERMT2 expression is dependent on miRNAs modulating APP metabolism}

We used an unbiased screening approach to identify miRNAs that modulate APP metabolism in a HCS model that allows for the quantification of intracellular APP fragments [14]. We screened a total of 2555 mature human miRNAs in a 384-well-plate format allowing us to identify 50 miRNAs (top and bottom 1\%) with the strongest impact on APP metabolism (Supplementary Table 2 and Supplementary Fig. 2). To determine which genes were potentially regulated by these top 50 hits, we selected the intersection of predictions resulting from at least four different algorithms (see "Methods") and thereby identified 6009 putative miRNA-target genes. To further refine the list of predicted genes, we cross-checked them against a list of 832 genes that we previously identified to have a major impact on APP metabolism in a genome-wide siRNA screening [14] (Supplementary Fig. 3). This resulted in 180 common genes that are putative targets of 41 miRNAs. To determine if any of these 180 genes were preferentially regulated by this pool of 41 miRNAs, we performed 1 million drawing lots of 41 miRNAs among the 2555 tested and compared them against the list of miRNAs predicted to bind in the $3^{\prime}$-UTR of each of the 180 genes (Supplementary Fig. 3). The AD genetic risk factor FERMT2 (encoding Kindlin-2) was among the most significant genes $\left(p\right.$ value $<2.77 \times 10^{-4}$ after Bonferroni correction) that strongly modulate APP metabolism, and whose expression is potentially regulated by miRNAs that also strongly modulate APP metabolism. According to our screening, four miRNAs were predicted to target FERMT2 $3^{\prime}$ UTR: miR-582-5p, miR-200b-3p, miR-221-3p, and miR-222-3p (Fig. 1a).

\section{miRNA-dependent FERMT2 expression and genetic variation associated with $A D$ risk}

Our data indicate that regulation of the FERMT2 expression is dependent on miRNAs, and we aimed to assess whether genetic variations associated with $\mathrm{AD}$ risk may modulate the miRNA-dependent expression of FERMT2. None of the variants localized within the FERMT2 $3^{\prime}$-UTR were predicted to modify the binding of miR-582-5p, miR-200b-3p, miR-221-3p, or miR-222-3p to this region (Supplementary methods). In contrast, we had previously identified an ADassociated variant (rs7143400), where the minor $\mathrm{T}$ allele creates an 8-mer binding site for miR-4504 within the $3^{\prime}$ UTR of FERMT2 [27] (Supplementary Fig. 4). This miRNA was not identified as a hit according to our screen, maybe due to the absence of rs 7143400-T allele in HEK293 cell line (Fig. 1c). Of note, there is a strong linkage disequilibrium between rs7143400 and the GWAS hit rs17125924 $\left(R^{2}=0.78\right)$, and rs7143400 has a lower minor allele frequency $(\mathrm{MAF}=0.09)$ and subsequently lower association $\left(p=7.14 \times 10^{-5}\right)$ than $\mathrm{rs} 17125924(\mathrm{MAF}=$ $\left.0.20, p=6.6 \times 10^{-7}\right)$. Supporting these predictions, we observed that miR-4504 led to reduced luciferase expression only in the presence of FERMT2 3'UTR rs7143400-T allele, whereas the four other miRNAs were able to induce a downregulation, regardless of the rs7143400 allele (Fig. 1b).

We then assessed the impact of these five miRNAs on endogenous FERMT2 expression levels after their transfection in either HEK293 ${ }^{\mathrm{WT}}$ or rs7143400-mutated HEK293 cell lines (HEK293 $3^{\text {r77143400-G/T }}$ ) generated by CRISPR-Cas9 technology (Fig. 1c and Supplementary Figs. 1 and 5). Accordingly, transfection of miR-582-5p, miR-200b-3p, miR-221-3p, or miR-222-3p in HEK293 cells led to reduced FERMT2 expression whatever their genotype, while transfection of miR-4504 decreased the endogenous FERMT2 expression only in the HEK293 $3^{\text {rs7143400-G/T }}$ cell line (Fig. 1d, e). Similar effects were observed in HeLa cells that were genotyped to be heterozygous for rs7143400 (Fig. 1c-e and Supplementary Fig. 5).

\section{Impact of miRNAs targeting FERMT2 on APP metabolism}

These five miRNAs are thus potential candidates to modulate APP metabolism through a direct downregulation of FERMT2. However, these miRNAs can also potentially target other genes strongly modulating APP metabolism (Supplementary Table 3). We reasoned that if a candidate miRNA affects APP metabolism mainly through 

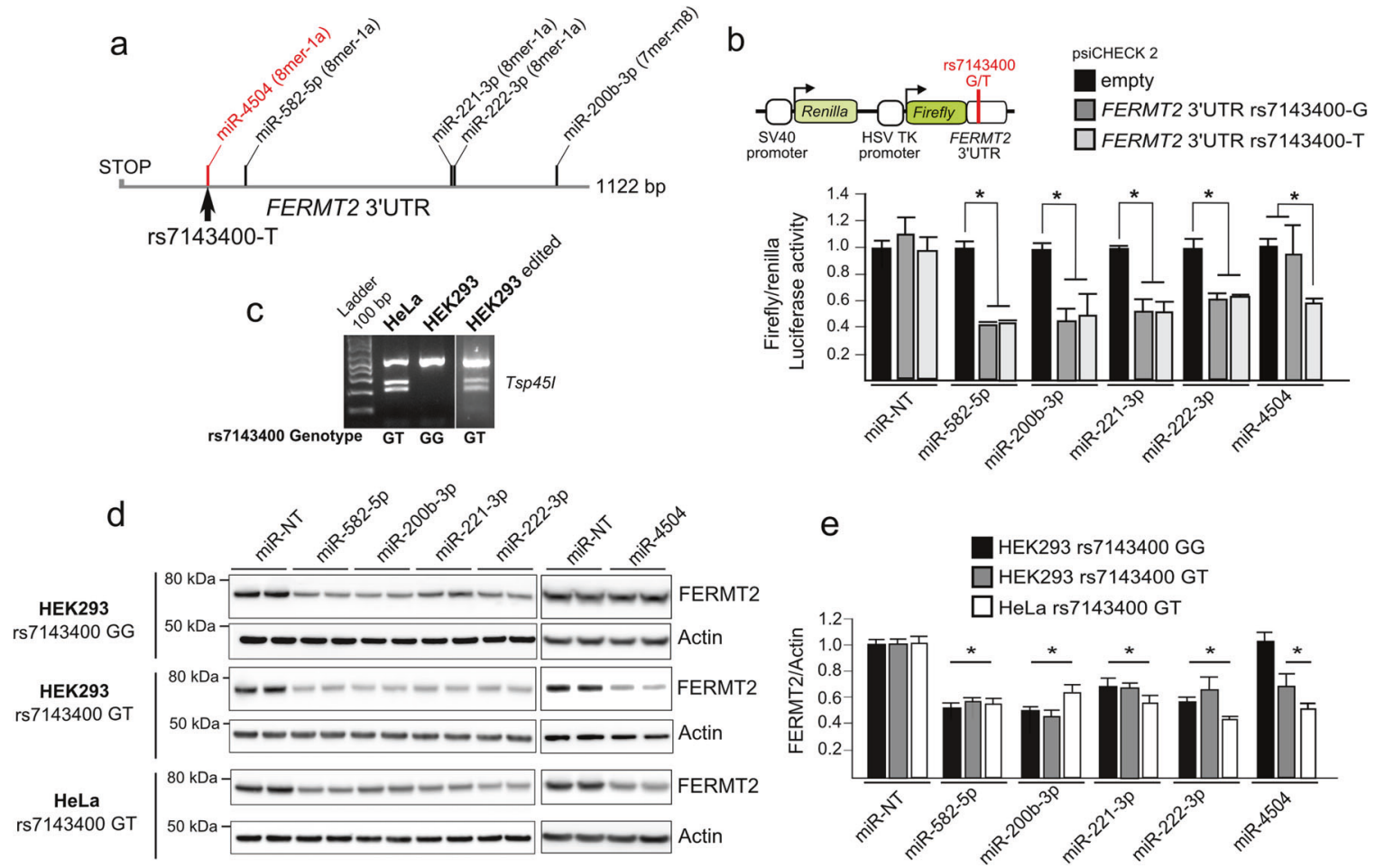

Fig. 1 Validation of functional miRNAs targeting FERMT2 $3^{\prime}$ UTR. a Relative positions of miRNA-target sites on FERMT2 3'UTR. The target site created by the rs7143400-T allele, which is associated with AD risk, is shown in red. b Luciferase activity of FERMT2 $3^{\prime}$ UTR carrying either the rs7143400-G or the rs7143400-T allele in HEK293 cells co-transfected with a nontargeting miRNA (miR-NT) or 5 miRNA mimics. Data are expressed relative to the miR-NT. $\mathbf{c}$ RFLP genotyping of HeLa and HEK293 cell lines edited or not for the

downregulating FERMT2, this candidate miRNA would have similar effects on APP metabolism as the direct FERMT2 downregulation by siRNAs we had previously demonstrated, i.e., leading to an increase in both intra- and extracellular by-products of APP [14]. To investigate this hypothesis, we used the data generated in our HCS approach (based on HEK293 cell line stably overexpressing a mCherry-APP695WT-YFP) in order to quantify intracellular by-products of APP $[14,28]$, and we also measured A $\beta$ and sAPP $\alpha$ secretion after miR-582-5p, miR-200b-3p, miR221-3p, or miR-222-3p transfections.

Only miR-582-5p and miR-222-3p showed similar effects as FERMT2 downregulation [14], i.e., they increased the levels of intracellular APP metabolites tagged by mCherrry and YFP, and increased $\mathrm{A} \beta$ and sAPP $\alpha$ secretion (Fig. 2a, b)

Since the potential effects of miR-4504 would depend on the presence of the rs7143400 minor T allele, we were not able to test for its impact in our HCS model. We nevertheless took advantage of HEK293 $3^{\text {rs7143400-G/T }}$ cells by cotransfecting them with miR-4504 and mCherry-APP ${ }^{695 \mathrm{WT}}$ YFP cDNA in order to mimic our HCS model. When rs7143400 via CRISPR-Cas9 (Supplementary Fig. 1). d Endogenous FERMT2 expression levels were assessed by Western blot using indicated cell extracts following transient transfection with a nontargeting miR (miR-NT) or with the indicated miR for $72 \mathrm{~h}$. e WB quantifications from three independent experiments as in $\mathbf{d}$ Data given in mean $\pm \mathrm{SD}$. $* p<0.05$, nonparametric test compared to miR-NT condition.

compared to HEK293 $3^{\mathrm{r} 57143400-\mathrm{G} / \mathrm{G}}$, the transfection of miR4504 in HEK293 $3^{\text {rs7143400-G/T }}$ led to an accumulation of intracellular APP mCherry and YFP-tagged metabolites and an increase in $A \beta$ and sAPP $\alpha$ secretion (Fig. 2c, d). MiR4504 showed similar effects as FERMT2 downregulation, and this observation further supports that miR-4504 regulates APP metabolism as a function of the FERMT2 rs7143400 variant.

In conclusion, we characterized that regulation of FERMT2 expression by miRNAs impacts APP metabolism, and potentially in a genetics-dependent manner.

\section{miRNA expression in different hippocampal cell types and in AD brains}

To provide further physiological relevance to our findings, we first combined classical immunocytochemistry with RNA hybridization that allows for the detection of miRNAs at single-copy sensitivity. We observed that miR-200, miR222, and miR-4504 were mainly expressed in neurons when compared to astrocytes (Fig. 3a). We next measured the expression levels of these miRNAs in the postmortem brain 


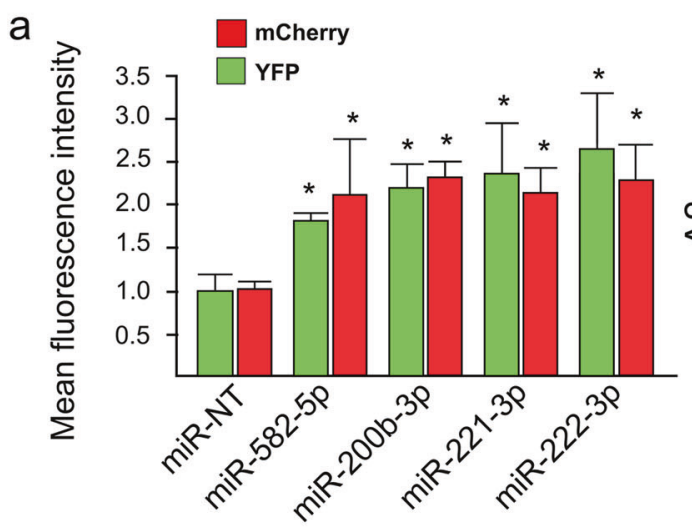

C

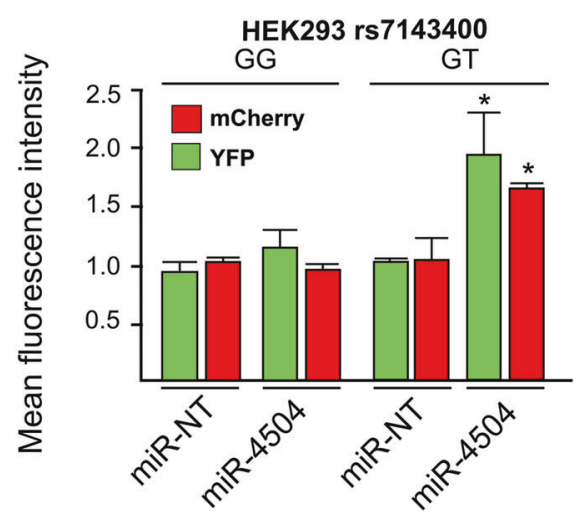

Fig. 2 Validation of the effects of FERMT2-targeting miRNA on APP metabolism. a Mean fluorescence intensity of intracellular mCherry and YFP signals obtained after miRNA transfection in HEK293 cells stably overexpressing a mCherry-APP ${ }^{695 \mathrm{WT}}$-YFP. b Quantification of $A \beta$ and sAPP $\alpha$ secretion after miRNA transfection in HEK293 cells stably overexpressing a mCherry-APP ${ }^{695 W T}$-YFP. c Mean fluorescence intensity variation of intracellular mCherry and

samples from $52 \mathrm{AD}$ patients and 30 control subjects. We observed that the expression levels of miR-200 and miR4504 were significantly higher in AD brains than in controls (Fig. 3b). Collectively, these data suggest that endogenous FERMT2 expression and its impact on APP metabolism are dependent on the expression of several miRNAs, two of which are overexpressed in the brains of AD cases and, among these two, one impacts APP metabolism in the presence of a genetic variant associated with AD risk.

\section{Pathway analyses suggest FERMT2/APP interaction to be involved in axonal growth}

Little is known about the physiological processes that require the regulation of APP expression and/or its metabolism by miRNAs. To obtain a list of potential physiological pathways to be further investigated, pathway-enrichment analysis was performed using the 41 candidate miRNAs that strongly modified APP metabolism in our HCS (Supplementary Table 2). This analysis revealed that the candidate miRNAs

b

d
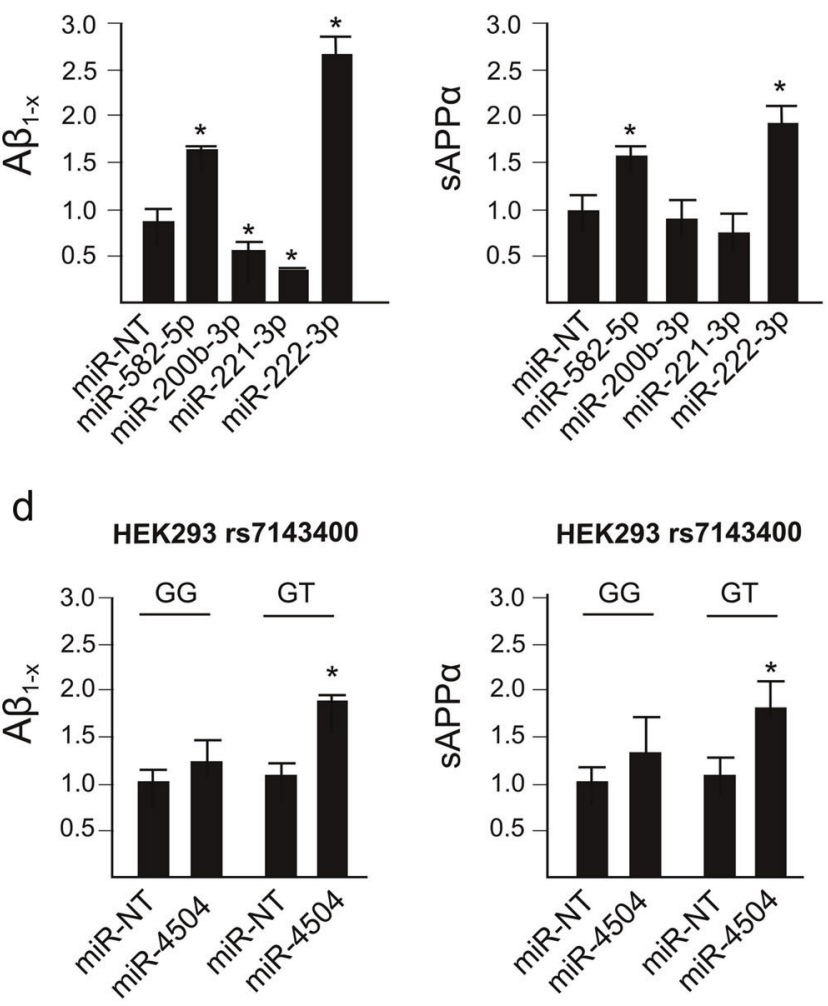

YFP signal obtained after miRNA transfection in HEK293 $3^{\text {rs7143400-G/G }}$ or HEK293 ${ }^{\text {rs7143400-G/T }}$ cell lines transiently overexpressing a mCherryAPP ${ }^{695 \mathrm{WT}}$-YFP. d Quantification of $\mathrm{A} \beta$ and $\mathrm{SAPP} \alpha$ secretion after miRNA transfection in HEK293 $3^{\text {rs7143400-G/G }}$ or HEK293 ${ }^{\text {rs7143400-G/T }}$ cell lines transiently overexpressing a mCherry-APP ${ }^{695 W T}$-YFP. Bar charts show mean \pm SD. Mann-Whitney test; $* p<0.05$.

are predicted to regulate neuronal pathways, such as axonal guidance (Table 1a). Since these 41 miRNAs potentially target 180 genes that strongly modulate APP metabolism (Supplementary Table 3), we also performed pathwayenrichment analysis using these 180 genes. This analysis revealed that these genes are predicted to be involved in axonal guidance among others (Table 1b). In conclusion, both miRNAs and genes modulating APP metabolism, e.g., FERMT2, potentially play a role in axonal guidance.

APP is already known to be enriched in axonal growth cones during nervous system development and acts as a coreceptor for axon guidance and cell migration cues through its interaction with the extracellular matrix $[29,30]$. We thus investigated the potential involvement of FERMT2 in axonal growth. Using primary neurons cultured in microfluidic devices that fluidically isolate axons from their cell bodies, we first observed the colocalization of endogenous FERMT2 with APP in the growth cones (Fig. 4a). We then addressed the impact of FERMT2 silencing on axonal growth cone morphology using lentiviral vectors expressing 

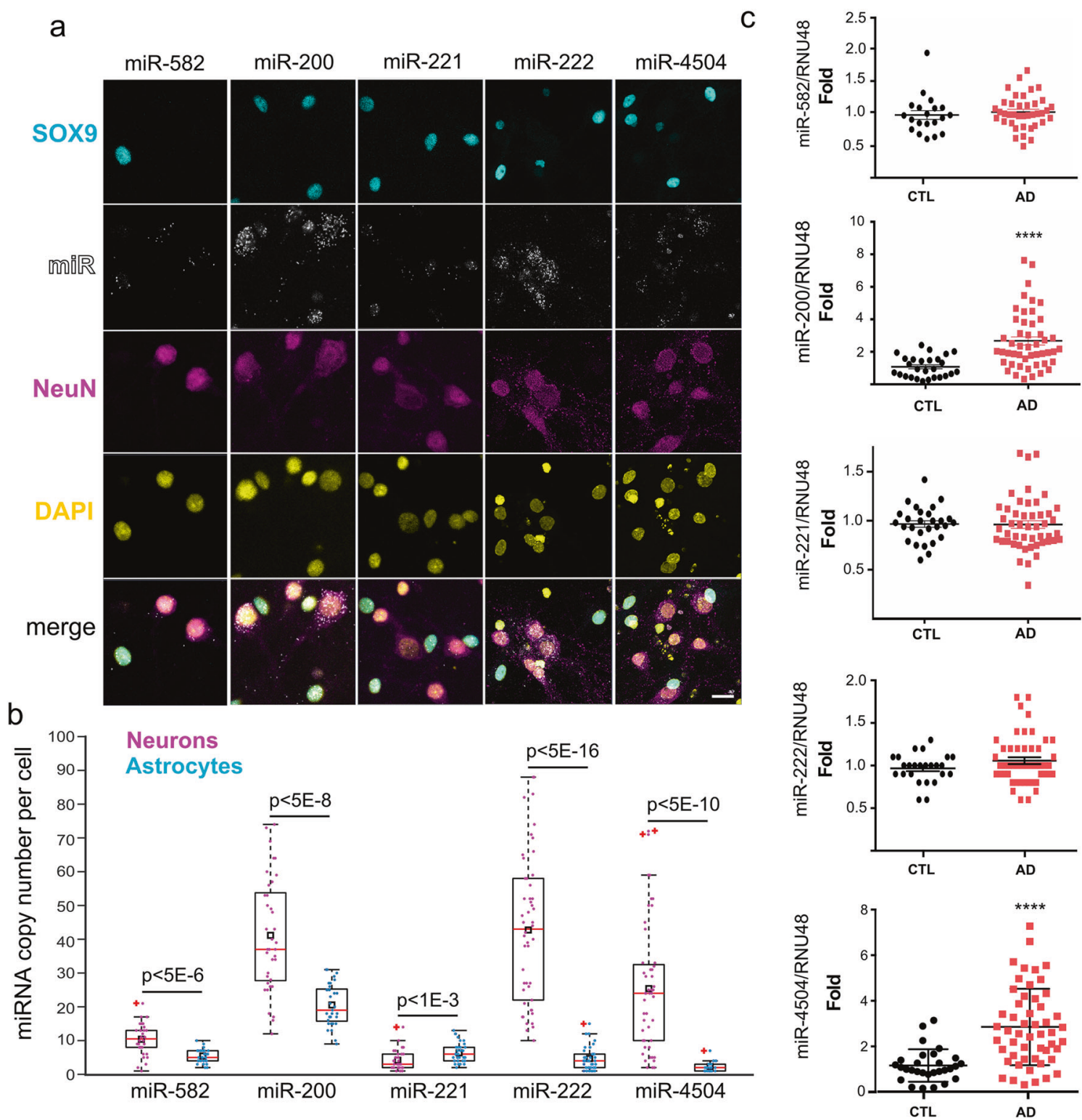

Fig. 3 miRNA expression in primary neuronal cultures and in AD brains. a Hybridization experiments in rat postnatal hippocampal neuronal cultures enabling single-copy detection of miRNA combined with immunocytochemistry against astrocytic (SOX9) and neuronal (NeuN) markers. Scale bar $=20 \mu \mathrm{m}$. The box plot shows the quantification of miRNA copy number in SOX9- or NeuN-positive cells $(N>30$ cells for each condition). Black rectangles and red plus signs indicate sample mean and outliers, respectively. b Relative miRNA expression levels in temporal lobes of nondemented (CTL) and AD groups. Mann-Whitney test; $* * * * p<0.0001$. either shRNA against FERMT2 (shFERMT2) or a nontargeting shRNA (shNT). Actin staining revealed that FERMT2 underexpression led to a significant decrease in growth cone area $\left(9.13 \pm 0.71\right.$ vs. $\left.12.79 \pm 1.10 \mu \mathrm{m}^{2}\right)$, as well as in the angular dispersion of growth cone filopodia during axonal growth $(0.67 \pm 0.04$ vs. $0.84 \pm 0.02)$ (Fig. $4 \mathrm{~b}$ and Supplementary Fig. 6). Of note, no significant impact on actin-retrograde flow rate was observed $(0.166 \pm 0.003$ vs. $0.157 \pm 0.002 \mu \mathrm{m} / \mathrm{s}$ ). These observations suggest a potential impairment of the exploration behavior of the growth cones due to FERMT2 silencing, but not an effect on actin dynamics per se. FERMT2 underexpression was also associated with an accumulation of endogenous APP in the growth cones $(1.38 \pm 0.11$ vs. $0.85 \pm 0.08$, after normalization by the growth cone area).

By performing PLA, we observed PLA-FERMT2/APP signals in axonal growth cones (Fig. 4a), suggesting a potential function of the FERMT2/APP complex in axongrowth behavior. To address this, we first tested the possibility that APP and FERMT2 form a protein-protein complex via three complementary approaches: (i) pull down of endogenous APP from hippocampal primary neuronal 
Table 1 Results of pathway-enrichment analyses.

\begin{tabular}{lcc}
\hline KEGG pathway & $p$ value & \#miRNAs \\
\hline (a) The ten most likely canonical pathways identified after pathway- \\
enrichment analysis of 41 miRNAs that strongly modulate APP \\
metabolism using DIANA Tools mirPath (v3.0) \\
Axon guidance & $4.70 \mathrm{E}-08$ & 48 \\
Proteoglycans in cancer & $1.82 \mathrm{E}-06$ & 49 \\
Hippo-signaling pathway & $4.10 \mathrm{E}-06$ & 50 \\
Fatty-acid biosynthesis & $8.10 \mathrm{E}-05$ & 11 \\
Glutamatergic synapse & $8.56 \mathrm{E}-05$ & 48 \\
GABAergic synapse & $1.10 \mathrm{E}-04$ & 48 \\
AMPK-signaling pathway & $2.20 \mathrm{E}-04$ & 46 \\
TGF-beta-signaling pathway & $2.50 \mathrm{E}-04$ & 45 \\
Thyroid hormone-signaling pathway & $3.90 \mathrm{E}-04$ & 48 \\
Adrenergic signaling in cardiomyocytes & $5.00 \mathrm{E}-04$ & 49 \\
KEGG pathway & $\boldsymbol{p}$ value & \#genes
\end{tabular}

(b) The ten most likely canonical pathways identified after pathwayenrichment analysis of 132 genes targeted by 41 miRNAs (see Supplementary Methods for details)

$\begin{array}{lll}\text { Axon guidance } & 0.0014 & 19 \\ \text { Ubiquitin-mediated proteolysis } & 0.010 & 16 \\ \text { Circadian rhythm } & 0.036 & 8\end{array}$

culture extracts co-immunoprecipitated endogenous FERMT2 (Fig. 4c). (ii) Overexpression of FERMT2 ${ }^{\mathrm{WT}}$ was also able to pull down the recombinant intracellular domain of APP (Fig. 4d). (iii) In addition, we generated a $\mathrm{Q}_{621} \mathrm{~W}_{622} \mathrm{AA}$ FERMT2 mutant $\left(\mathrm{FERMT2}{ }^{\mathrm{QW}}\right.$ ) that was previously shown to abolish the interaction between the FERMT2 F3 domain and the NxTY motif of Integrin- $\beta 3$ (which is also present within the intracellular domain of APP) [31]. Remarkably, when overexpressed in HEK293 cells, FERMT2 ${ }^{\mathrm{QW}}$ was not able to pull down the recombinant intracellular domain of APP. Cumulatively, these findings support a direct interaction between FERMT2 and APP. Based on the recently solved crystal structure of FERMT2 in complex with the integrin- $\beta 3$ tail [32], we built a structural model of the FERMT2/APP complex (Fig. 4e), supporting our hypothesis that a protein-protein interaction exists between FERMT2 and APP.

We next assessed the biological impact of the FERMT2/ APP interaction on APP metabolism. By performing extracellular biotinylation experiments, we observed that FERMT2 overexpression in HEK293-APP ${ }^{695 \mathrm{wt}}$ cell line decreased the levels of APP at the cell surface, an effect that was abolished by the presence of the QW mutation (Fig. 4f). Further, a dominant negative effect of the FERMT2 ${ }^{\mathrm{QW}}$ mutant was observed: its overexpression impacted APP metabolism similarly to FERMT2 silencing, i.e., resulting in increased mature APP at the cell surface and increased A $\beta$ production, as previously reported [14]. Altogether, our data suggest that a FERMT2/APP interaction is necessary for FERMT2 to have an impact on APP metabolism.

In order to characterize in-depth the impact of FERMT2 and/or APP expression on axonal growth, we conducted time-lapse microscopy and measured axon-growth speed at DIV5 following lentiviral transduction (shNT, shFERMT2, or shAPP) of neurons in microfluidic devices at DIV1. FERMT2 silencing led to $31.7 \%$ increase in axon-growth speed (Fig. 5 and Supplementary Fig. 6). Conversely, APP underexpression led to $16.7 \%$ decrease in axon-growth speed. Remarkably, silencing of APP was able to fully abolish the effect of FERMT2 underexpression on axongrowth speed, suggesting that APP was required for the molecular mechanism by which FERMT2 controls the axon-growth speed. In addition, we observed that FERMT2 ${ }^{\mathrm{QW}}$ mutant overexpression was able to induce $15.9 \%$ increase in axon-growth speed (Fig. 5). Since overexpression of FERMT2 ${ }^{\mathrm{WT}}$ did not show any impact, these data also suggested a potential dominant negative effect of the FERMT2 ${ }^{\mathrm{QW}}$ mutant and further supported the involvement of FERMT2/APP complex in axonal growth.

\section{FERMT2 is present at the synapse and controls synaptic connectivity}

Next, we investigated the impact of FERMT2 silencing on neuronal maturation at DIV14. First, co-staining between FERMT2 and synaptic markers (Synaptophysin and Homer) suggested the localization of FERMT2 at the synapse (Fig. 6a). The presence of FERMT2 in both pre- and postsynaptic compartments was confirmed by synaptosomal purification (Fig. 6b). To control shRNA expression separately in pre- or postsynaptic neurons, hippocampal neurons were cultured in microfluidic devices that promote synapse formation in an isolated chamber [33]. Thanks to the use of narrow microchannels, these devices spatially isolate neurites from their cell bodies and allow lentiviral transductions to be conducted in different compartments, thereby allowing us to silence FERMT2 expression at the pre- and/or postsynaptic levels (Fig. 6c and Supplementary Fig. 6). The effects of shRNA expression (DIV1) on synaptic connectivity were assessed by confocal microscopy of synaptic markers (DIV14) followed by three-dimensional image segmentation and quantification [26]. Underexpression of FERMT2 in the presynaptic chamber led to a decrease in synaptic connectivity, whereas no such effect was observed when underexpressing FERMT2 in the postsynaptic compartment (Fig. 6d). Altogether, our data suggest that FERMT2 expression is required for synapse connectivity. Moreover, PLA-FERMT2/APP signals were colocalized with Synaptophysin and Homer puncta (Fig. 6e), supporting the possibility of the involvement of the FERMT2/APP complex in synapses. 
a
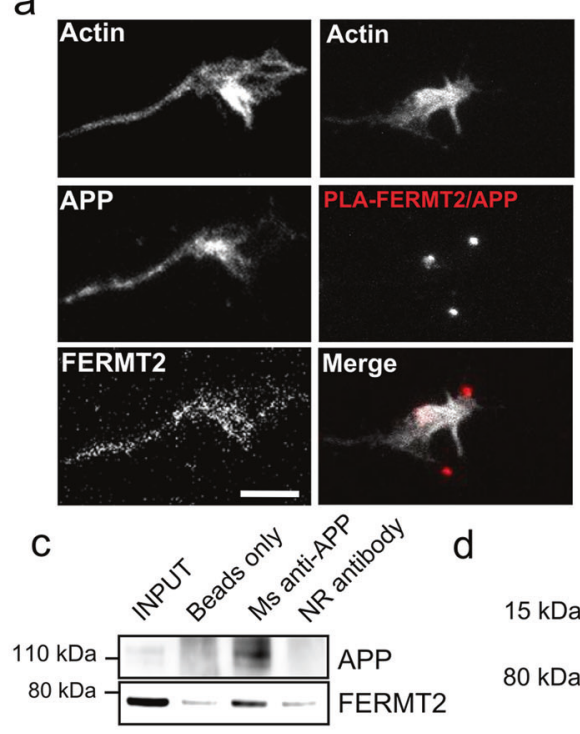

d

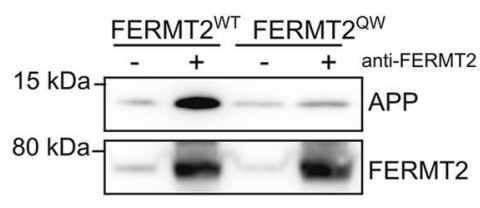

shNT

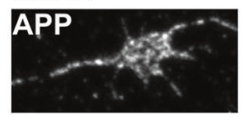

shFERMT2

APP
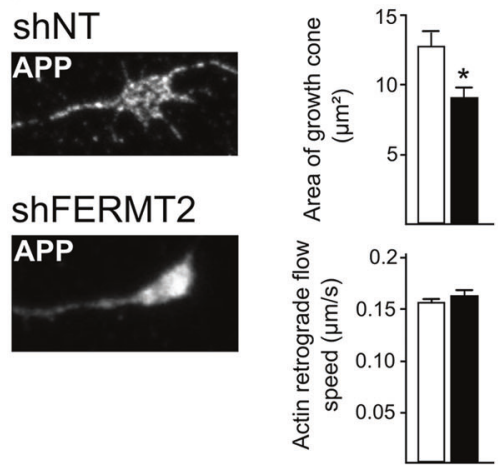

e
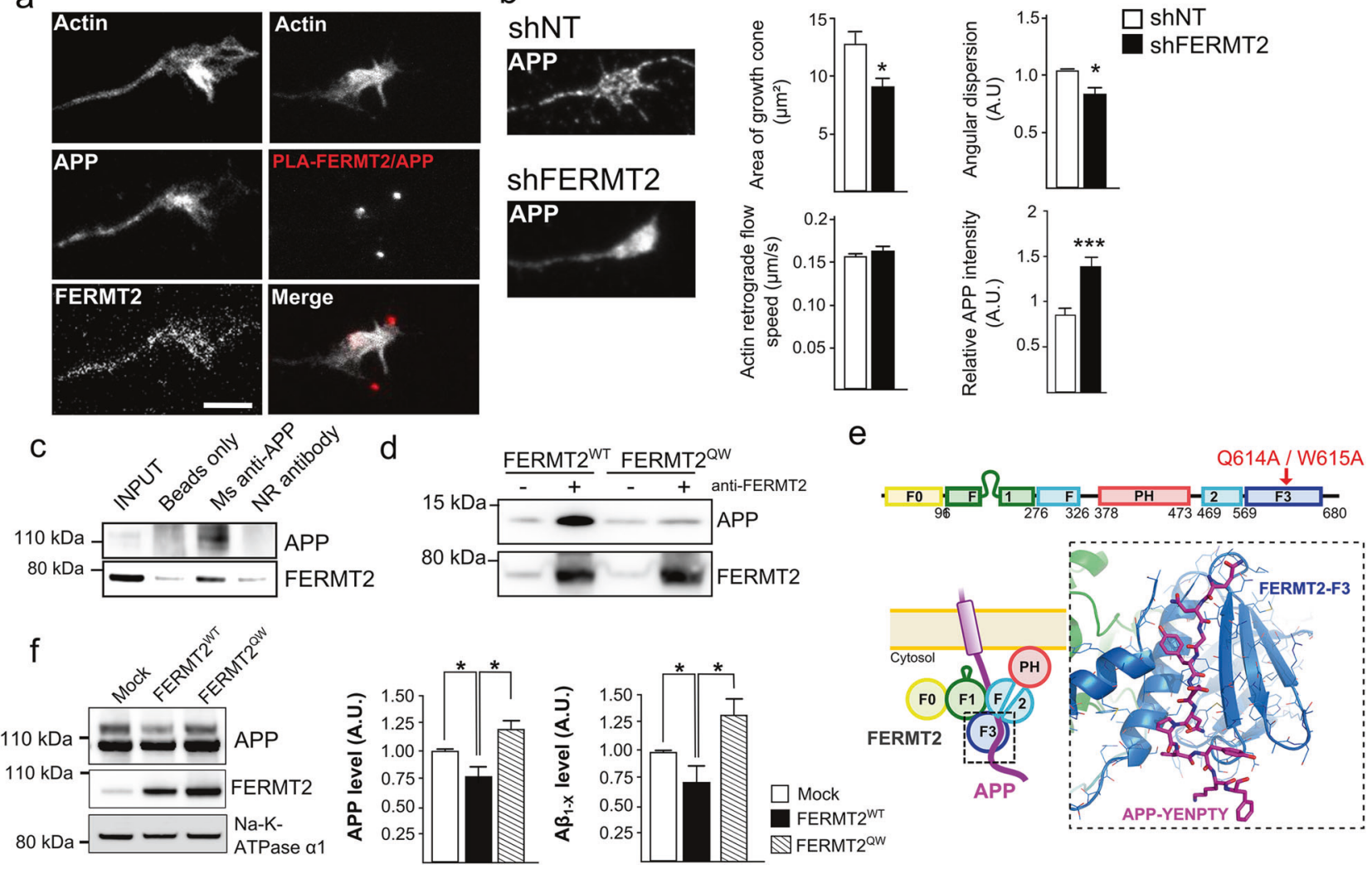

type (WT) or mutated (QW) FERMT2. Protein extracts from HeLa

Fig. 4 FERMT2 directly interacts with APP. a Immunofluorescence images showing the presence of APP and FERMT2 within the axonal growth cone stained with SiR-Actin. The right panel shows the presence of PLA-FERMT2/APP puncta within the axonal growth cone. b Impact of lentiviral transduction of nontargeting shRNA (shNT) or shRNA against FERMT2 (shFERMT2) on growth cone area, angular dispersion and speed of the actin-retrograde flow, and APP immunostaining. c Co-IP between endogenous APP and FERMT2 from membrane extracts of hippocampal PNC. Protein extracts were incubated with beads only, a mouse (Ms) antibody against APP (4G8), or a nonrelevant (NR) antibody. d APP pull-down experiment with wild-

\section{FERMT2 expression regulates synaptic plasticity in an APP-dependent manner}

We sought to establish the functional impact of FERMT2 and/or APP silencing on PPF and LTP in ex vivo mouse (10-week-old male) hippocampal slices, after stereotactic lentivirus injection allowing for the expression of shNT, shFERMT2, shAPP, or shFERMT2+shAPP.

Broadly speaking, PPF arises due to increased presynaptic $\mathrm{Ca}^{2+}$, which leads to the release of neurotransmitter in two distinct waves. In this situation, two action potentials in the presynaptic cell produce two excitatory postsynaptic potentials (EPSPs) in the postsynaptic cell: the first action potential produces the first EPSP, but the second action potential produces an EPSP that is larger than the EPSP produced by the first. PPF modulation therefore highlights a modulation in presynaptic neurotransmitter release [34]. Using this readout cells overexpressing FERMT2 ${ }^{\mathrm{WT}}$ or FERMT ${ }^{\mathrm{QW}}$ were incubated with recombinant APP C-terminal fragment (C100). e The domain organization of FERMT2 protein (upper panel). Q614A/W615A (QW) mutation was reported to abolish the interaction of F3 domain of FERMT2 with the NxTY motif. The structural model of the FERMT2APP complex (lower panel) was built by homology using the crystal structure of the FERMT2-Integrin- $\beta 3$-tail complex [32]. f The impact of FERMT2 on APP metabolism in HEK293-APP ${ }^{695 W T}$ cells is reverted with the overexpression of FERMT2 ${ }^{\mathrm{QW}}$ compared to FERMT2 ${ }^{\mathrm{WT}}$. Scale bar $=5 \mu \mathrm{m}$. Mann-Whitney test; $* p<0.05$.

as a proxy for presynaptic function, we observed a significant decrease in PPF in shFERMT2-infected mice compared to shNT-infected control mice (Fig. 7a). This PPF impairment however was rescued when APP was also downregulated (shAPP + shFERMT2 group).

In separate experiments, tetanic stimulation was delivered to the Shaffer collaterals (SC) in order to induce LTP in hippocampal slices (Fig. 7b). Tetanic stimulation of the SC resulted in a robust, long-lasting potentiation of the fEPSP slope in slices from mice infected with shNT and with shAPP, whereas LTP was impaired in slices from shFERMT2infected mice. This suggests that an LTP deficit was observed in hippocampal slices infected with shFERMT2, but not in those infected with shAPP (Fig. 7c, d). Remarkably, this deficit was abolished when both APP and FERMT2 were silenced, suggesting that APP was required for the molecular mechanism by which FERMT2 impacts LTP. 


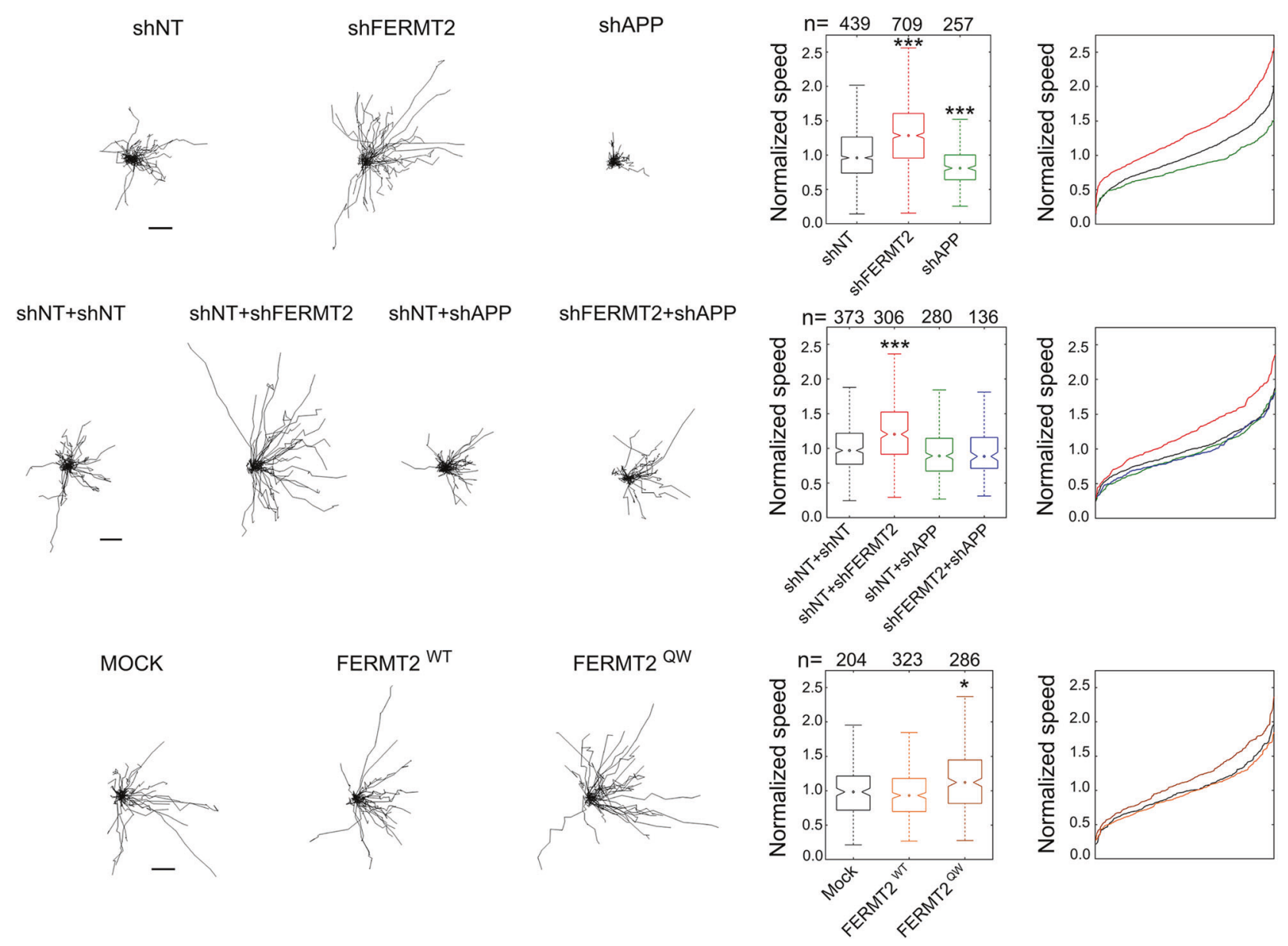

Fig. 5 FERMT2 regulates axonal growth rate depending on APP expression. Impact of lentiviral transduction on axonal growth speed. Individual axon tracks from a representative set are plotted. Scale bar $=50 \mu \mathrm{m}$. Box plots and cumulative-distribution plots are color-

Importantly, in these slices, no significant difference was observed for the normalized average slope of the evoked fEPSP, indicating that no alteration of the CA1 basal synaptic transmission occurred in any of the groups analyzed (Fig. 7e and Supplementary Fig. 7).

Altogether, these data are in agreement with our previous observations that FERMT2 is involved in the presynaptic compartment and modulates synaptic connectivity in an APP-dependent manner.

\section{Discussion}

As in other multifactorial diseases, GWAS in AD are agnostic approaches, and how a genetic risk factor is implicated in pathophysiological processes is typically unknown. Sometimes, even the physiological functions of an $\mathrm{AD}$ genetic risk factor in the brain are not known. Understanding the role of these genes is thus a challenge that requires several key questions to be addressed: (i) does matched. $n$ is the number of axons analyzed from at least three independent experiments. Kruskal-Wallis ANOVA with multiple comparisons; $* p<5 \times 10^{-3} ; * * * p<5 \times 10^{-7}$.

the corresponding protein interact (directly or indirectly) with other key players and pathways known to be involved in AD? (ii) What is (are) the functional variant(s) responsible for the GWAS signal and does this (do these) variant (s) impact the biological function of the corresponding protein and its interaction with key players of $\mathrm{AD}$ ?

To answer these questions, we developed systematic approaches to determine the genes that are involved in APP metabolism, a major player in $\mathrm{AD}$ development. To this end, we had previously developed a HCS, based on the quantification of intracellular APP fragments, to measure the impact of underexpression of 18,107 genes (via siRNA pools) on APP metabolism [14]. In the current study, we screened the impact of the overexpression of 2555 miRNAs on APP metabolism with the hypothesis that genes (i) that modulate the APP metabolism and (ii) whose expression levels are regulated by miRNAs that also modulate the APP metabolism are likely some of the key actors controlling the APP metabolism and functions. The convergence of these two agnostic screens highlighted FERMT2, a GWAS-defined genetic risk 
a
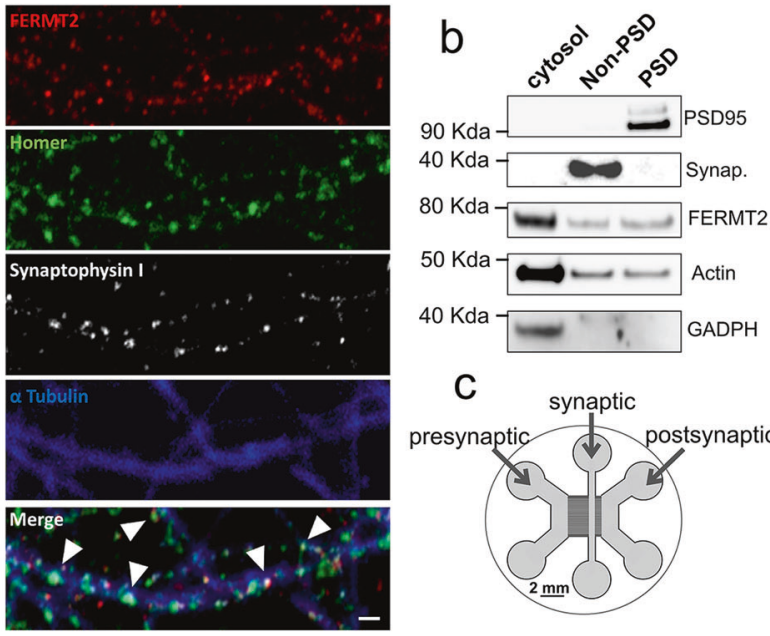

\section{C}

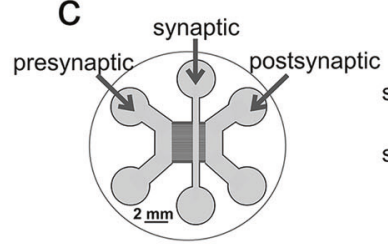

Fig. 6 FERMT2 is present at the synapse and controls synaptic connectivity. a Immunofluorescence in hippocampal primary neuronal culture showing the colocalization of FERMT2 puncta with pre- and postsynaptic markers, Synaptophysin and Homer, respectively. b Synaptic fractionation experiment revealed the presence of FERMT2 in both pre- and postsynaptic compartments. c Schematics of the tricompartmental microfluidic device. The use of microchannels with different lengths ensures that only axons arrive from the presynaptic to the synaptic compartment, where synapses can be observed

factor of $\mathrm{AD}$, for which almost nothing is known in the cerebral and $\mathrm{AD}$ contexts.

We demonstrated that a direct interaction between FERMT2 and APP-through the F3 domain of FERMT2 and the NXTY motif within APP's intracellular domain-is necessary for FERMT2 to have an impact on APP metabolism. Moreover, we observed that the FERMT2/APP interaction could be involved in the regulation of axonal growth, in line with APP's function within the growth cone [30] (data we replicated in this study). It has been reported that FERMT2 is required for the recruitment and activation of focal adhesion kinase and the triggering of integrin signaling [15]. In neurons, the focal adhesion pathway is involved in synaptic density and activity through regulating the dendritic spine shape, stability, and the signaling machinery therein [35]. That is why we also analyzed synaptic plasticity, a readout highly relevant to $\mathrm{AD}$, where synaptic dysfunction/loss is one of the earliest events observed. FERMT2 underexpression had detrimental effects on PPF (presynaptic) and LTP (postsynaptic). Remarkably, in both cases, the detrimental effect of FERMT2 underexpression was dependent on APP expression. In this context, it is important to note that numerous evidence indicate that presynaptic physiological functions involving APP, which has been recently proposed as a structural and functional regulator of the hippocampal presynaptic active zone [36], could be major molecular players in $\mathrm{AD}$ [37]. As FERMT2 silencing leads to an accumulation of full-length APP and all its by-products (including A $\beta$ peptides), we can
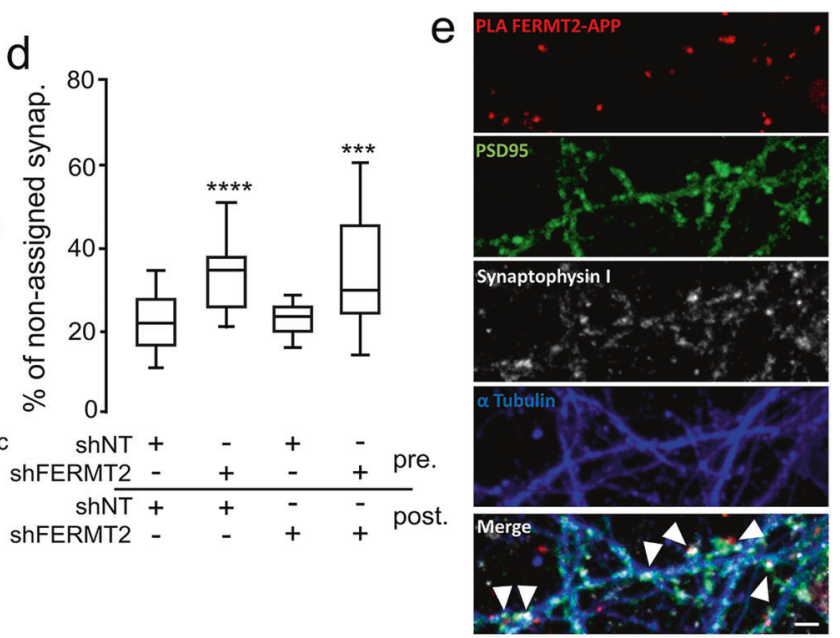

independently of the cell bodies. The device also permits lentiviral transductions to be performed exclusively in the pre- and postsynaptic compartments. d Synaptic connectivity as a function of FERMT2 underexpression in pre- and postsynaptic chambers. Increased fraction of Synaptophysin spots not assigned by a Homer spot within a distance threshold of $1 \mu \mathrm{m}$ is indicative of decreased synaptic connectivity. e PLA-FERMT2/APP puncta were observed at the synapses stained for pre- and postsynaptic markers. Scale bar $=2 \mu \mathrm{m}$.

hypothesize that these accumulations could be involved in the synaptic dysfunction observed due to FERMT2 underexpression, although further experiments are needed to decipher the potential causal link between FERMT2 and APP, i.e., to determine whether FERMT2 impacts the function of full-length APP or invokes A $\beta$ synaptotoxicity. This is of particular interest, since APP shedding strongly enhances its cell adhesion and synaptogenic activity [38]. Moreover, APP's intracellular domain is required for normal synaptic morphology and plasticity, suggesting that its intracellular interaction partners could be required for proper synaptic function [39]. Remarkably, we have recently proposed a circular model of $\mathrm{AD}$ pathogenesis, where the core of the focal adhesion pathway-in which FERMT2 and APP are part of-may participate in the dysfunction of synaptic plasticity in AD [13].

We have also identified that FERMT2 expression level is highly regulated by miRNAs that could be preferentially expressed in neurons. In addition, we previously identified the rs7143400 variant located in FERMT2 3'UTR to be susceptible to alter a binding site for miR-4504 [27]. Here, in addition to in silico prediction suggesting the impact of this variant on miRNA binding (Supplementary Fig. 4), we demonstrate that this variant is functional: the $\mathrm{AD}$-associated rs7143400-T allele downregulates FERMT2 and modulates APP metabolism via its interaction with miR-4504. Remarkably, we observed that miR-4504 is overexpressed in the brains of $\mathrm{AD}$ cases compared to controls, and is mainly expressed in neurons in primary mixed hippocampal cultures. 
a

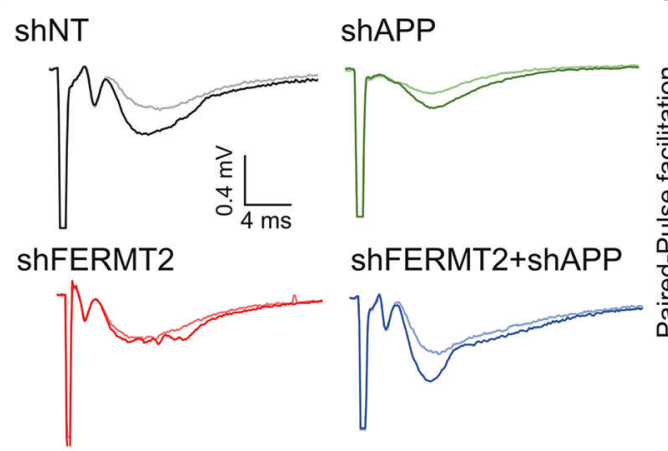

C

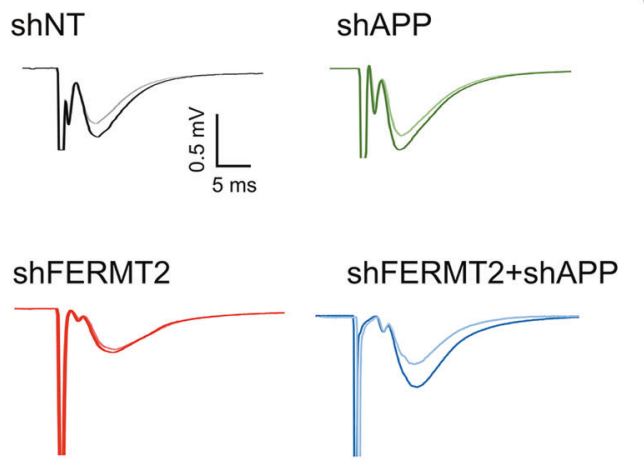

e

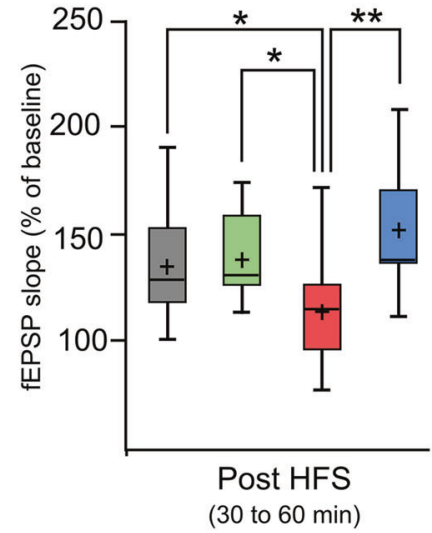

Fig. 7 FERMT2 underexpression alters PPF and LTP in an APPdependent manner. a Paired-pulse facilitation (PPF) as a function of the interstimulus interval 7 days after viral injection of indicated lentivirus. $n=3$ mice, 2 slices per animal. b Exemplary fEPSP traces during baseline (light line) and 30-60 min after LTP induction (dark line). $\mathbf{c}$ Time course of the average slope of elicited fEPSP responses following LTP induction by a tetanic stimulation protocol in hippocampal CA1 synapses after viral injection. Time point 0 represents the delivery of the tetanic stimulation. Slopes of each fEPSP are

Together, our data indicate that a deleterious overexpression of miR-4504 can lead to a decrease in FERMT2 expression in individuals bearing the rs7143400 minor T allele, which subsequently modulates APP metabolism. Interestingly, similar mechanism has been reported for genetic variants associated with $\mathrm{AD}$ risk in APP $3^{\prime} \mathrm{UTR}$ that regulates APP expression b
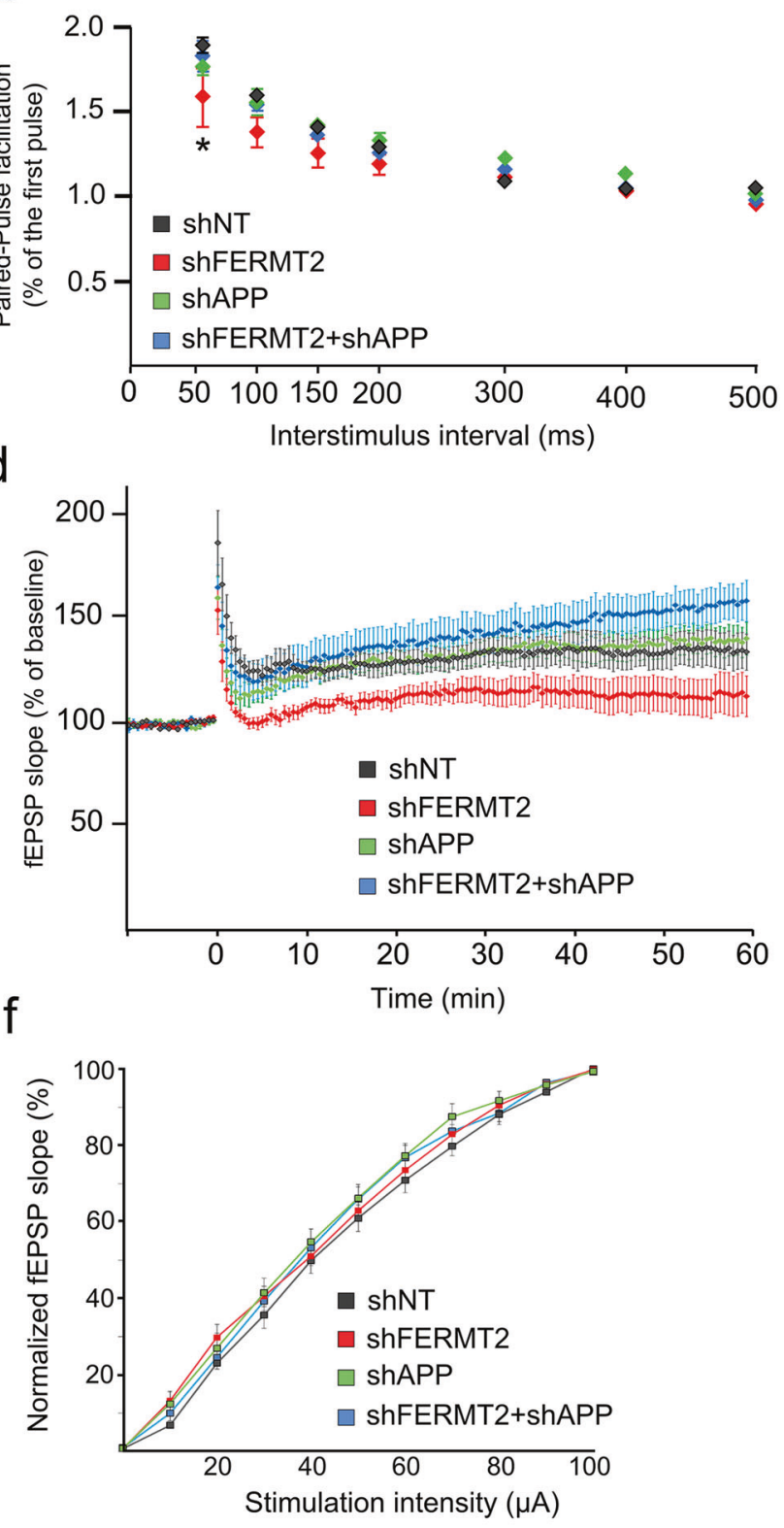

normalized by the baseline and plotted against time. $\mathbf{d}$ Box plots of the average slope response during 30-60 min post LTP induction. HFS high-frequency stimulation. $n=5$ mice, two slices per animal. e Normalized average slope of fEPSP evoked in hippocampal slices from animals injected with the indicated lentivirus. Recordings have been performed in the stratum radiatum of hippocampal CA1 region with electrical stimulation of Schaffer collaterals (see "Methods"). Unpaired $t$ test; ${ }^{*} p<0.05 ; * * p<0.01$.

through miRNA binding [40]. Supporting a link between FERMT2 and APP metabolism, studies from cohorts of patients have reported an association between variants in FERMT2 gene and A $\beta$ in CSF [14] and brain amyloidosis [41].

Here, we propose that FERMT2 downregulation at the earliest stage of AD would depend in part on (i) the miR-4504 
expression, (ii) cerebral cell type (i.e., neurons), and (iii) the presence of the rs7143400 minor T allele (observed in 9\% of Caucasians). Unfortunately, it is important to keep in mind that all these constraints will make difficult, if not impossible, to detect such a miRNA-dependent decrease in FERMT2 mRNA levels. Of note, this point may also underline the limitation of expression databases in deciphering the mechanisms underlying the functional effects of GWAS variants, for they do not allow capturing (even hiding) subtle mechanisms.

In publicly available RNA-seq analyses (Mayo Clinic Brain Bank), an overexpression of FERMT2 mRNA has been observed in postmortem human temporal cortex of $\mathrm{AD}$ patients relative to healthy controls [42]. Even though a small sample size did not allow us to observe such a variation in FERMT2 mRNA levels, we nevertheless detected an increase in FERMT2 protein levels as a function of Braak stage, especially at later stages (Supplementary Fig. 8). This point is of particular importance since in the Genotype-Tissue Expression Database [43], FERMT2 variants associated with an increase in AD risk at the genomewide significance level are also part of an expression quantitative trait locus, significantly associated with decreased brain expression of FERMT2 mRNA (sentinel variant in GWAS rs17125924; $-18 \% ; p$ value $=2 \times 10^{-6}$ ). Altogether, these results strongly support the notion that FERMT2 downregulation is deleterious at the earliest stages of the disease, whereas FERMT2 overexpression may occur as a pathological consequence at a later stage. In this context, it would be of interest to further investigate the impact of FERMT2 downregulation in vivo. Since the loss of FERMT2 in mice results in embryonic lethality at E7.5 [44], a dedicated mouse model should be developed that allows conditional FERMT2 knockout in neurons. This model would then be crossed with AD-like mouse models to assess behavioral and $\mathrm{AD}$-associated phenotypes.

In conclusion, we propose that FERMT2 underexpression through miRNAs and/or genetic regulation leads to synaptic dysfunction in an APP-dependent manner. Our hypothesis may thus call for new therapeutic approaches in AD targeting FERMT2 and/or APP function.

Acknowledgements FE benefited from a doctoral grant co-funded by Hauts-de-France Regional Council and Institut Pasteur de Lille. The authors thank the BICeL and EquipEx ImagInEx HCS platforms of the Institut Pasteur de Lille and Alexandre Vandeputte for technical assistance. The authors thank Karine Blary at the IEMN Lille for the microfabrication work. This work was partly supported by the French RENATECH network. This study was funded by INSERM, Institut Pasteur de Lille, the Centres of Excellence in NeurodegenerationANR program (CoEN, GWAS in AD: focus on microRNA), the Canadian Institute of Health Research (CHIR), la Fondation Alzheimer (Syn-Alz), the EU Joint Programme-Neurodegenerative Diseases Research (JPND, 3DMiniBrain), and Fondation Vaincre Alzheimer (FR-17006p). This work was also funded by the Lille Métropole Communauté Urbaine and the French government's LABEX DISTALZ program (development of innovative strategies for a transdisciplinary approach to Alzheimer's disease). This work was supported for MH by Academy of Finland (Grant Number 307866), Sigrid Jusélius Foundation, and the Strategic Neuroscience Funding of the University of Eastern Finland. The authors thank the vectorology platform Transbiomed for lentivirus production.

Author contributions J-CL and JC designed and supervised research. AF, FE, FC, and CB performed APP metabolism and FERMT2/APP interaction analyses. XH developed the in silico model for FERMT2/ APP interaction. FE and DK performed and analyzed axon-growth experiments. AC, AF, JD, and JC developed Crispr/Cas9 model and/or performed subsequent analyses. $\mathrm{CD}, \mathrm{A}-\mathrm{CV}, \mathrm{AF}$, and $\mathrm{BG}-\mathrm{B}$ designed and/or performed miRNA screening and/or statistical analyses. SH and MF performed and analyzed electrophysiology experiments. AF, TM, $\mathrm{FD}$, and SD performed primary neuronal cultures. MM, MT, IP, and $\mathrm{MH}$ analyzed transcriptomic and/or proteomic data of FERMT2 expression in brains. EB and SSH performed miR expression quantification in brains. FE, NM, DK, and JC participated in image acquisition and analyses of APP/FERMT2 interaction and/or synapse density. FE, PA, JD, DK, J-CL, and JC wrote and/or revised the paper.

\section{Compliance with ethical standards}

Conflict of interest SH and MF are full-time employees of E-PhyScience SA. CD has been an employee of Janssen Pharmaceutica since her departure from the laboratory Inserm U1167 in 2016.

Publisher's note Springer Nature remains neutral with regard to jurisdictional claims in published maps and institutional affiliations.

Open Access This article is licensed under a Creative Commons Attribution 4.0 International License, which permits use, sharing, adaptation, distribution and reproduction in any medium or format, as long as you give appropriate credit to the original author(s) and the source, provide a link to the Creative Commons license, and indicate if changes were made. The images or other third party material in this article are included in the article's Creative Commons license, unless indicated otherwise in a credit line to the material. If material is not included in the article's Creative Commons license and your intended use is not permitted by statutory regulation or exceeds the permitted use, you will need to obtain permission directly from the copyright holder. To view a copy of this license, visit http://creativecommons.org/licenses/by/4.0/.

\section{References}

1. Checler F. Processing of the beta-amyloid precursor protein and its regulation in Alzheimer's disease. J Neurochem. 1995;65:1431-44.

2. Hardy J. Amyloid, the presenilins and Alzheimer's disease. Trends Neurosci. 1997;20:154-9.

3. Hardy J, Selkoe DJ. The amyloid hypothesis of Alzheimer's disease: progress and problems on the road to therapeutics. Science. 2002;297:353-6.

4. Morris GP, Clark IA, Vissel B. Questions concerning the role of amyloid- $\beta$ in the definition, aetiology and diagnosis of Alzheimer's disease. Acta Neuropathol. 2018;136:663-89.

5. Jonsson T, Atwal JK, Steinberg S, Snaedal J, Jonsson PV, Bjornsson $\mathrm{S}$, et al. A mutation in APP protects against Alzheimer's disease and age-related cognitive decline. Nature. 2012;488:96-9.

6. Nicolas G, Charbonnier C, Wallon D, Quenez O, Bellenguez C, Grenier-Boley B, et al. SORL1 rare variants: a major risk factor for familial early-onset Alzheimer's disease. Mol Psychiatry. 2016;21:831-6. 
7. Andersen OM, Reiche J, Schmidt V, Gotthardt M, Spoelgen R, Behlke J, et al. Neuronal sorting protein-related receptor sorLA/ LR11 regulates processing of the amyloid precursor protein. Proc Natl Acad Sci USA. 2005;102:13461-6.

8. Raghavan N, Tosto G. Genetics of Alzheimer's disease: the importance of polygenic and epistatic components. Curr Neurol Neurosci Rep. 2017;17:78.

9. Kim J, Basak JM, Holtzman DM. The role of apolipoprotein E in Alzheimer's disease. Neuron. 2009;63:287-303.

10. Jay TR, Hirsch AM, Broihier ML, Miller CM, Neilson LE, Ransohoff RM, et al. Disease progression-dependent effects of TREM2 deficiency in a mouse model of alzheimer's disease. $\mathbf{J}$ Neurosci. 2017;37:637-47.

11. Jansen IE, Savage JE, Watanabe K, Bryois J, Williams DM, Steinberg S, et al. Genome-wide meta-analysis identifies new loci and functional pathways influencing Alzheimer's disease risk. Nat Genet. 2019;51:404-13.

12. Kunkle BW, Grenier-Boley B, Sims R, Bis JC, Damotte V, Naj $\mathrm{AC}$, et al. Genetic meta-analysis of diagnosed Alzheimer's disease identifies new risk loci and implicates $A \beta$, tau, immunity and lipid processing. Nat Genet. 2019;51:414-30.

13. Dourlen P, Kilinc D, Malmanche N, Chapuis J, Lambert J-C. The new genetic landscape of Alzheimer's disease: from amyloid cascade to genetically driven synaptic failure hypothesis? Acta Neuropathol. 2019;138:221-36.

14. Chapuis J, Flaig A, Grenier-Boley B, Eysert F, Pottiez V, Deloison $\mathrm{G}$, et al. Genome-wide, high-content siRNA screening identifies the Alzheimer's genetic risk factor FERMT2 as a major modulator of APP metabolism. Acta Neuropathol. 2017;133:955-66.

15. Theodosiou M, Widmaier M, Böttcher RT, Rognoni E, Veelders M, Bharadwaj M, et al. Kindlin-2 cooperates with talin to activate integrins and induces cell spreading by directly binding paxillin. Elife. 2016;5:e10130.

16. Dowling JJ, Gibbs E, Russell M, Goldman D, Minarcik J, Golden JA, et al. Kindlin-2 is an essential component of intercalated discs and is required for vertebrate cardiac structure and function. Circ Res. 2008;102:423-31.

17. Shen Z, Ye Y, Dong L, Vainionpää S, Mustonen H, Puolakkainen $\mathrm{P}$, et al. Kindlin-2: a novel adhesion protein related to tumor invasion, lymph node metastasis, and patient outcome in gastric cancer. Am J Surg. 2012;203:222-9.

18. Zhan J, Zhu X, Guo Y, Wang YY, Wang YY, Qiang G, et al. Opposite role of kindlin-1 and kindlin-2 in lung cancers. PLoS ONE. 2012;7:1-12.

19. Sossey-Alaoui K, Pluskota E, Szpak D, Plow EF. The Kindlin2p53-SerpinB2 signaling axis is required for cellular senescence in breast cancer. Cell Death Dis. 2019;10:539.

20. Blasiak A, Lee GU, Kilinc D. Neuron subpopulations with different elongation rates and DCC dynamics exhibit distinct responses to isolated Netrin-1 treatment. ACS Chem Neurosci. 2015;6:1578-90.

21. Kaech S, Banker G. Culturing hippocampal neurons. Nat Protoc. 2006;1:2406-15.

22. Smith PY, Delay C, Girard J, Papon M-A, Planel E, Sergeant N, et al. MicroRNA-132 loss is associated with tau exon 10 inclusion in progressive supranuclear palsy. Hum Mol Genet. 2011;20:4016-24.

23. Sevalle J, Amoyel A, Robert P, Fournié-Zaluski M-C, Roques B, Checler F. Aminopeptidase A contributes to the N-terminal truncation of amyloid beta-peptide. J Neurochem. 2009;109:248-56.

24. Meijering E, Dzyubachyk O, Smal I. Methods for cell and particle tracking. Methods Enzymol. 2012;504:183-200.

25. Frandemiche ML, De Seranno S, Rush T, Borel E, Elie A, Arnal I, et al. Activity-dependent tau protein translocation to excitatory synapse is disrupted by exposure to amyloid-Beta oligomers. J Neurosci. 2014;34:6084-97.
26. Kilinc D, Vreulx A-C, Mendes T, Flaig A, Marques-Coelho D, Verschoore M, et al. Pyk2 overexpression in postsynaptic neurons blocks amyloid $\beta 1-42$-induced synaptotoxicity in microfluidic cocultures. Brain Commun. 2020. https://doi.org/10.1093/bra incomms/fcaa139.

27. Delay C, Grenier-Boley B, Amouyel P, Dumont J, Lambert J-C. miRNA-dependent target regulation: functional characterization of single-nucleotide polymorphisms identified in genome-wide association studies of Alzheimer's disease. Alzheimers Res Ther. 2016;8:20.

28. Sannerud R, Declerck I, Peric A, Raemaekers T, Menendez G, Zhou L, et al. ADP ribosylation factor 6 (ARF6) controls amyloid precursor protein (APP) processing by mediating the endosomal sorting of BACE1. Proc Natl Acad Sci U S A. 2011;108:E559-68.

29. Soldano A, Hassan BA. Beyond pathology: APP, brain development and Alzheimer's disease. Curr Opin Neurobiol. 2014;27:61-7.

30. Sosa LJ, Bergman J, Estrada-Bernal A, Glorioso TJ, Kittelson JM, Pfenninger KH. Amyloid precursor protein is an autonomous growth cone adhesion molecule engaged in contact guidance. PLoS ONE. 2013;8:e64521.

31. Ma Y-Q, Qin J, Wu C, Plow EF. Kindlin-2 (Mig-2): a co-activator of beta3 integrins. J Cell Biol. 2008;181:439-46.

32. Li H, Deng Y, Sun K, Yang H, Liu J, Wang M, et al. Structural basis of kindlin-mediated integrin recognition and activation. Proc Natl Acad Sci USA. 2017. https://doi.org/10.1073/pnas.1703064114.

33. Taylor AM, Dieterich DC, Ito HT, Kim SA, Schuman EM. Microfluidic local perfusion chambers for the visualization and manipulation of synapses. Neuron. 2010;66:57-68.

34. Gebhardt C, Mosienko V, Alenina N, Albrecht D. Priming of LTP in amygdala and hippocampus by prior paired pulse facilitation paradigm in mice lacking brain serotonin. Hippocampus. 2019;29:610-8.

35. Hotulainen P, Hoogenraad CC. Actin in dendritic spines: connecting dynamics to function. J Cell Biol. 2010;189:619-29.

36. Weingarten J, Weingarten M, Wegner M, Volknandt W. APP-A novel player within the presynaptic active zone proteome. Front Mol Neurosci. 2017;10:43.

37. Barthet G, Mulle C. Presynaptic failure in Alzheimer's disease. Prog Neurobiol. 2020;194:101801.

38. Stahl R, Schilling S, Soba P, Rupp C, Hartmann T, Wagner K, et al. Shedding of APP limits its synaptogenic activity and cell adhesion properties. Front Cell Neurosci. 2014;8:410.

39. Klevanski M, Herrmann U, Weyer SW, Fol R, Cartier N, Wolfer DP, et al. The APP intracellular domain is required for normal synaptic morphology, synaptic plasticity, and hippocampusdependent behavior. J Neurosci. 2015;35:16018-33.

40. Nicolas G, Wallon D, Goupil C, Richard A-C, Pottier C, Dorval $\mathrm{V}$, et al. Mutation in the 3'untranslated region of APP as a genetic determinant of cerebral amyloid angiopathy. Eur J Hum Genet. 2016;24:92-8.

41. Apostolova LG, Risacher SL, Duran T, Stage EC, Goukasian N, West JD, et al. Associations of the top 20 alzheimer disease risk variants with brain amyloidosis. JAMA Neurol. 2018;75:328.

42. Sullivan SE, Liao M, Smith RV, White C, Lagomarsino VN, Xu J, et al. Candidate-based screening via gene modulation in human neurons and astrocytes implicates FERMT2 in A $\beta$ and TAU proteostasis. Hum Mol Genet. 2018. https://doi.org/10.1093/hmg/ ddy376.

43. GTEx Consortium KG, Deluca DS, Segre AV, Sullivan TJ, Young TR, Gelfand ET, et al. Human genomics. The GenotypeTissue Expression (GTEx) pilot analysis: multitissue gene regulation in humans. Science. 2015;348:648-60.

44. Dowling JJ, Vreede AP, Kim S, Golden J, Feldman EL. Kindlin-2 is required for myocyte elongation and is essential for myogenesis. BMC Cell Biol. 2008;9:36. 